\title{
Exotic see-saw mechanism for neutrinos and leptogenesis in a Pati-Salam model
}

\author{
Andrea Addazi, ${ }^{a, b}$ Massimo Bianchi ${ }^{c}$ and Giulia Ricciardi ${ }^{d, e}$ \\ ${ }^{a}$ Dipartimento di Fisica, Università di L'Aquila, \\ 67010 Coppito, AQ, Italy \\ ${ }^{b}$ LNGS, Laboratori Nazionali del Gran Sasso, \\ 67010 Assergi AQ, Italy \\ ${ }^{c}$ Dipartimento di Fisica, Università di Roma Tor Vergata, \\ I.N.F.N. Sezione di Roma Tor Vergata, \\ Via della Ricerca Scientifica, 100133 Roma, Italy \\ ${ }^{d}$ Dipartimento di Fisica "E. Pancini", Università di Napoli "Federico II", \\ Complesso Universitario di Monte Sant'Angelo, Via Cintia, 80126 Napoli, Italy \\ ${ }^{e}$ I.N.F.N. Sezione di Napoli, \\ Complesso Universitario di Monte Sant'Angelo, Via Cintia, 80126 Napoli, Italy \\ E-mail: andrea.addazi@infn.lngs.it, massimo.bianchi@roma2.infn.it, \\ giulia.ricciardi@na.infn. it
}

ABSTRACT: We discuss non-perturbative corrections to the neutrino sector, in the context of a D-brane Pati-Salam-like model, that can be obtained as a simple alternative to $\mathrm{SO}(10)$ GUT's in theories with open and unoriented strings. In such D-brane models, exotic stringy instantons can correct the right-handed neutrino mass matrix in a calculable way, thus affecting mass hierarchies and modifying the see-saw mechanism to what we name exotic see-saw. For a wide range of parameters, a compact spectrum of right-handed neutrino masses can occur that gives rise to a predictive scenario for low energy observables. This model also provides a viable mechanism for Baryon Asymmetry in the Universe (BAU) through leptogenesis. Finally, a Majorana mass for the neutron is naturally predicted in the model, leading to potentially testable neutron-antineutron oscillations. Combined measurements in neutrino and neutron-antineutron sectors could provide precious informations on physics at the quantum gravity scale.

KEYWORDS: Strings and branes phenomenology

ARXIV EPRINT: 1510.00243 


\section{Contents}

1 Introduction 1

2 Pati-Salam-like D-brane models $\quad 5$

$\begin{array}{llr}2.1 & \text { Free parameters } & 10\end{array}$

$\begin{array}{lll}2.1 .1 & \text { Supersymmetry and string scale } & 10\end{array}$

$\begin{array}{ll}\text { 2.1.2 Relevant effective Lagrangian and free parameters } & 10\end{array}$

3 Phenomenology in neutrino physics $r$

$\begin{array}{ll}3.1 \text { Conditions for a compact RH neutrino spectrum } & 11\end{array}$

$\begin{array}{lll}3.2 & \text { Low energy observables } & 13\end{array}$

4 Leptogenesis $\quad 16$

$\begin{array}{llr}5 & \text { Phenomenology in neutron-antineutron physics } & 19\end{array}$

6 Conclusions and remarks $\quad 20$

\section{Introduction}

In [1], Majorana proposed the existence of extra mass terms of the form $m \psi \psi+h . c$, in which $\psi$ is a neutral fermion, such as a neutrino or a neutron. Majorana's proposal has never seemed to be so up-to-date and intriguing as today. In fact, from several measures of atmospheric, solar, accelerator and reactor neutrinos, neutrino oscillations have been fully confirmed. These observations represent evidence that neutrinos are massive. Majorana's proposal goes even beyond the mass issues: a Majorana mass term for neutrinos or for the neutron leads to violation of Lepton (L) and Baryon (B) numbers as $\Delta L=2$ and $\Delta B=2$, respectively. The Standard Model (SM) does not offer an adequate explanation of the observed Matter-Antimatter asymmetry in our Universe, i.e. the SM does not generate the necessary Lepton and/or Baryon number asymmetries in the primordial Universe. The possibility of a Majorana mass term for neutrino or neutron can disclose new paths towards the origin of the observed asymmetry and its possible dynamical generation, through a viable mechanism for baryogenesis.

See-saw Type I mechanism is considered one of the most elegant ways to explain the observed smallness of neutrino masses [2-6]. In see-saw Type I, right-handed (RH) neutrinos with masses much higher than the electroweak (EW) scale are required. Remarkably, this mechanism offers a simple and natural solution for leptogenesis, a model of baryogenesis where the lightest $\mathrm{RH}$ neutrino can decay into lighter particles [7]. In the primordial universe, near the EW phase transition, leptons, quarks and Higgs also interact via $B+L$ violating non-perturbative interactions, generated by sphalerons, leading to an effective 
conversion of part of the initial lepton number asymmetry into a baryonic one [15]. Moreover, the complex Yukawa couplings of the RH neutrinos can provide new sources of $\mathrm{CP}$ violation. All Sakharov's conditions to dynamically generate baryon asymmetry [8] are satisfied: 1) out of thermal equilibrium condition; 2) CP violations; 3) baryon number violation. The sphaleron-mediated effective interactions were calculated for the first time by t'Hooft [14]. These effects are strongly suppressed in our present cosmological epoch but, in the primordial thermal bath, they are expected to be unsuppressed, leading to non-negligible corrections to the chemical potentials.

The see-saw mechanism can be naturally embedded in a Pati-Salam (PS) model $\mathrm{SU}(3)_{c} \times \mathrm{SU}(2)_{L} \times \mathrm{SU}(2)_{R} \times \mathrm{U}(1)_{B-L}$ or $\mathrm{SU}(4)_{c} \times \mathrm{Sp}(2)_{L} \times \mathrm{Sp}(2)_{R}$ [16]. As suggested in [6] Majorana masses for neutrinos can be elegantly connected to a spontaneous symmetry breaking of parity and to leptogenesis. In fact the RH masses are related to LeftRight scale and $\mathrm{U}(1)_{B-L} \subset \mathrm{SU}(4)_{c}$ spontaneous symmetry breaking scale. On the other hand, a RH neutrinos mass scale of order $M_{R} \sim 10^{9 \div 13} \mathrm{GeV}$ is necessary for consistent leptogenesis [27].

As a natural step beyond a PS-model, SO(10) GUT could unify the SM with U(1) $B-L$ via an intermediate $\mathrm{SU}(4)_{c} \times \mathrm{SU}(2)_{L} \times \mathrm{SU}(2)_{R}$ PS-like gauge group. ${ }^{1}$ However, let us recall that the $\mathrm{SO}(10)$ GUT scenario presents some challenging theoretical problems, that are generally solved at the cost of some complications of the initial GUT model. Problems such as proton destabilization and the imperfect unification of coupling constants are generally alleviated in SUSY SO(10) GUT. With or without SUSY, the most serious hierarchy problem for $\mathrm{SO}(10)$ and other GUTs is the doublet-triplet splitting. The standard Higgs doublet is contained in $10_{H}$ (or $5_{H}+5_{H}^{*}$ in $\mathrm{SU}(5)$ ), leading to dangerous scale-mixing diagrams between standard doublets and heavier Higgs triplets inside $10_{H}$. In other words, a stabilization of the ordinary doublet at much smaller scales than $M_{G U T} \simeq 10^{15 \div 16} \mathrm{GeV}$ is highly unnatural, i.e. it reintroduces another Higgs hierarchy problem even if one assumes 1 TeV SUSY breaking scale. ${ }^{2}$

In $\mathrm{SO}(10)$, the quark-lepton symmetry makes the reconciliation of leptogenesis and seesaw mechanism more problematic. In fact, assuming the spontaneous symmetry breaking scale of $\mathrm{SU}(4)_{c} \times \mathrm{SU}(2)_{L} \times \mathrm{SU}(2)_{R} \rightarrow \mathrm{SU}(3)_{c} \times \mathrm{SU}(2)_{L} \times \mathrm{U}(1)_{Y}$ around $\Lambda_{R} \simeq 10^{11} \mathrm{GeV}$, the lightest $\mathrm{RH}$ eigenstate $N_{1}$, which is generally the main responsible for generating a lepton asymmetry, acquires a mass $M_{R_{1}} \ll 10^{9} \mathrm{GeV}$. Unfortunately, this value is well below the Davidson-Ibarra (DI) bound [27] $\left(M_{D I} \gtrsim 10^{9} \mathrm{GeV}\right)$, guaranteeing a sufficient production of lepton asymmetry from $\mathrm{RH}$ neutrino decays. There are basically three ways out of this difficulty. One possibility is to consider leptogenesis where crucial contributions arise via the decays of heavier RH neutrinos, with masses above the DI limit [28-34]. Alternatively, one can assume a highly compact spectrum [35, 36]. Finally, in a situation in which one

\footnotetext{
${ }^{1}$ Recent discussions about $\mathrm{SO}(10)$ GUT can be found in [9-13].

${ }^{2}$ The doublet-triplet problem can be solved in different ways in GUT models. The most popular solution is the missing partner or vacuum-expectation-value mechanism for $\mathrm{SU}(5)$ [17]. In $\mathrm{SO}(10)$, an implementation of this mechanism was shown in [18]. As an alternative, we mention pseudo-Nambu-Goldstone boson mechanism for SU(6) [19-21]. Finally, in string theory (and orbifold GUTs), orbifold projection can remove Higgs triplets [22-26].
} 
pair of $\mathrm{RH}$ neutrinos is highly degenerate, the DI bound can be avoided through a resonant enhancement of CP asymmetries [37,38]. Let us observe that the latter two scenarios are not easily incorporated in $\mathrm{SO}(10) .^{3}$

Lastly, it is undoubtable that $\mathrm{SO}(10)$ cannot provide a way to unify gravity with the other interactions. Indeed, $\mathrm{SO}(10)$ scenarios are not the only possible completion of PS-like models. In IIA and IIB superstring theory, a natural way to construct a PS-like model can be achieved through a system of intersecting D-branes stacks wrapping some sub-manifold ('cycles') in a Calabi-Yau (CY) compactifications with open strings ending on them. In this class of models, a different kind of geometric unification can be achieved, including gravity - even if string theory were incomplete, even if quantum gravity were only understood partially. ${ }^{4}$ Recently, a simple D-branes PS-like model was suggested in [47]. In [47], we have noticed that a Higgs sector composed of $\Delta(10,1,1), \Delta^{c}\left(10^{*}, 1,1\right), \phi_{L L}(1,3,1), \phi_{R R}(1,1,3)$ and $h_{L R}(1,2,2)$, the latter containing SM Higgses, can reproduce the right pattern of fermion masses. However, the above Higgses cannot break $\mathrm{SU}(4) \times \mathrm{SU}(2)_{R}$ down to $\mathrm{SU}(3) \times \mathrm{U}(1)_{Y}$ in the desired way. This spontaneous symmetry breaking can be obtained through Higgs superfields $\bar{H}(\overline{4}, 1,2)$ and $H(4,1,2)$. In $\mathrm{SO}(10)$, they are usually contained in $16_{H}, \overline{16}_{H} \cdot \bar{H}$ has the same representation $F_{R}$ of the standard fermions and their super-partners, while $H$ is in the conjugate one. They can be decomposed in components as

$$
\begin{aligned}
& \bar{H}(\overline{4}, 1,2)=\left(u_{R}^{c}, d_{R}^{c}, e_{R}^{c}, \nu_{R}^{c}\right) \\
& H(4,1,2)=\left(\bar{u}_{R}^{c}, \bar{d}_{R}^{c}, \bar{e}_{R}^{c}, \bar{\nu}_{R}^{c}\right)
\end{aligned}
$$

The vacuum expectation values (VEVs) along the "sneutrino" components

$$
\langle\bar{H}\rangle=\left\langle\nu_{R}^{c}\right\rangle, \quad\langle H\rangle=\left\langle\bar{\nu}_{R}^{c}\right\rangle
$$

break $\mathrm{SU}(4) \times \mathrm{SU}(2)_{R}$ to $\mathrm{SU}(3) \times \mathrm{U}(1)_{Y}$. VEVs $(1.3)$ have to be higher than $\left\langle\Delta^{c}\right\rangle,\left\langle\phi_{R R}\right\rangle$ in order to guarantee the right symmetry breaking pattern. ${ }^{5}$ In this model a Majorana mass for the neutron and extra terms in the $\mathrm{RH}$ neutrinos mass matrix are generated by Euclidean D2-branes (or E2-branes), wrapping a different 3-cycle with respect to the ordinary D6-branes. Such E2's are called exotic instantons. They are a different kind of instantons not present in gauge theories. The effect of E2s are calculable and controllable in models like our one. Unlike 'gauge' instantons, 'exotic' instantons do not admit an

\footnotetext{
${ }^{3}$ For recent literature discussing these aspects, see [40-42].

${ }^{4}$ As in GUTs, also in these models we can find some difficult theoretical problems: i) the identification of the precise CY singularity for the D-brane construction, ii) the quantitative stabilization of geometric moduli for the particular realistic particle physics model considered. These problems are expected to be solved by including fluxes and the effects of stringy instantons. For the moment, awaiting for a more precise quantitative UV completion (global embedding) of our model, we can neglect these problematics. Our attitude is to consider effective string-inspired models, locally free from anomalies and tadpoles and interesting for phenomenology of particle physics and cosmology. On the other hand, attempts to solve the problems mentioned above are the main topics of an intense investigation. For example, see [44-46] for recent discussions.

${ }^{5}$ For this reason, a TeV-ish Left-Right symmetry breaking is not favored by our precise model. Comments on phenomenological aspects made in [47] can be valid in quivers inspired by the present one but with extra nodes.
} 
ADHM construction. See $[49,50]$ for useful reviews of these aspects. ${ }^{6}$ The main new peculiar feature of exotic instantons is that they can violate vector-like symmetries like baryon and lepton numbers! B/L-violations by exotic E2-instantons are not necessarily suppressed: suppression factors depend on the particular size of the 3-cycles wrapped in the CY compactification by exotic E2-instantons. A dynamical violation of a symmetry is something "smarter" than an explicit one: all possible dangerous operators are not generated by exotic instantons, only few interesting operators can be generated. For instance, an effective operator $\left(u^{c} d^{c} d^{c}\right)^{2} / \Lambda_{n \bar{n}}^{5}$ is generated in our model, without proton destabilization: a residual discrete symmetry is preserved by exotic instantons, avoiding $\Delta B=1$ processes but allowing $n-\bar{n}(\Delta B=2)$ transitions [43]. In particular, such transitions are mediated by three color scalar sextets present in our model. E2-instantons generate an effective superpotential term $\mathcal{W}_{E 2}=\Delta_{u^{c} u^{c}}^{(6)} \Delta_{d^{c}{ }^{c}}^{(6)} \Delta_{d^{c} d^{c}}^{(6)}\left\langle S^{(1)}\right\rangle / \mathcal{M}_{E}$, where $\Delta_{6}=(6,1)_{+2 / 3}$ and $S=(1,1)_{-2}$ are contained in $(10,1,1)$ of $\mathrm{SU}(4)_{c} \times \mathrm{Sp}(2)_{L} \times \mathrm{Sp}(2)_{R}$. When $S$ takes an expectation value, spontaneously breaking $\mathrm{U}(1)_{B-L}$, an effective trilinear interaction for $\Delta^{(6)} \mathrm{S}$ is generated at low energies of order $\mathcal{M}_{E} \sim M_{S}$, where $M_{S}$ is the string scale. $n-\bar{n}$ transition can be obtained from $\mathcal{W}_{E 2}$ and renormalizable operators, present in our model and coded in a quiver, $\Delta_{u^{c} u^{c}}^{(6)} u^{c} u^{c}$ and $\Delta_{d^{c} d^{d}}^{(6)} d^{c} d^{c}$, with $\Lambda_{n \bar{n}}^{5} \simeq \mathcal{M}_{E} M_{\Delta_{u^{c} u^{c}}}^{2} M_{\Delta_{d^{c} d^{c}}}^{2} M_{\mathrm{SUSY}} / v_{B-L}$ where $M_{\mathrm{SUSY}}$ is the SUSY breaking scale, $v_{B-L}$ the $\mathrm{U}(1)_{B-L}$ breaking VEV. Its scale can be as low as $\Lambda \simeq 1000 \mathrm{TeV}$, corresponding to $n-\bar{n}$ transitions in vacuum (no magnetic-fields, outside nuclei) with $\tau_{n \bar{n}} \simeq 100 \mathrm{yr}$, i.e. $10^{-33} \tau_{p \text {-decay }}$ [52]. The next generation of experiments promises to test exactly this scale, enhancing the current best limits for $\tau_{n \bar{n}}$ [53] by two orders of magnitude [54-56]. In string theory, $M_{S}$ needs not be necessarily close to the Planck scale, it can easily stay at a lower scale. Similarly the SUSY breaking scale is not necessarily at the TeV scale - since we are only interested in SUSY as a symmetry for superstring theory, we will consider it to be around the String scale. ${ }^{7}$ Direct limits on color sextet scalars can be obtained from FCNCs as discussed in [60, 61], usually stronger than LHC ones $[62,63] .{ }^{8}$ In the present paper, we discuss quantitative predictions of our PS-like model for low energy observables in neutrino physics, as done in the literature for $\mathrm{SO}(10)$ GUT's. We show that our model can be remarkably predictive for neutrino physics, exposing a quark-lepton symmetry and a compact spectrum of RH neutrinos with masses above the DI bound for leptogenesis. The compactness of the mass spectrum of RH neutrinos is related to the geometrical proprieties of the relevant mixed disk amplitudes. Our model provides a theoretical framework where a compact RH spectrum emerges naturally. In our phenomenological analysis, we will take into account a non vanishing value of the lepton mixing angle $\theta_{13}$, as measured in [71-73], assuming the best fit value given in [73]. We will

\footnotetext{
${ }^{6}$ See [51] for a recent paper on D-brane instantons in chiral quiver theories.

${ }^{7}$ An alternative mechanism for Baryon Asymmetry of the Universe (BAU) can be envisaged. As proposed in [43, 57-59], a Post-Sphaleron Baryogenesis mediated by color scalar sextets could be a viable alternative to a Leptogenesis-Sphaleron mechanism. An intriguing possibility is to test this scenario in NeutronAntineutron physics. Color scalar sextets are naturally embedded not only in $\mathrm{SO}(10)$, but also in our model with intersecting D-branes, as extensively discussed in [47].

${ }^{8}$ For other D-branes model generating a Majorana mass for the neutron and other intriguing signatures for phenomenology, in Ultra Cold Neutron Physics, Ultra High Energy Cosmic Rays, FCNCs and LHC, see [64-70].
} 
see how the compactness of the RH neutrinos mass spectrum leads to consistent solution with a non-zero Dirac phase $\delta \neq 0$, in the Pontecorvo-Maki-Nagakawa-Sakata (PMNS) mixing matrix. The solutions obtained then fix the other unknown low energy parameters: the PMNS CP violating phases $\delta, \alpha, \beta$ (modulo signs) and the left-handed (LH) neutrino mass scale $M_{1}$. We also predict the RH neutrino masses. The numerical approach follows the path drawn in the context of $\mathrm{SO}(10) \mathrm{GUT}$, where a compact $\mathrm{RH}$ spectrum represented a somewhat arbitrary assumption $[35,36]$. The plan of the paper is as follows. In section 2 we review and amend a Pati-Salam-like model with gauge $\mathrm{U}(4) \times \operatorname{Sp}(2)_{L} \times \operatorname{Sp}(2)_{R}$ based on unoriented D-branes proposed in [47]. In section 4 we calculate relevant parameters for leptogenesis in a case where the right order of magnitude and sign of the BAU is recovered, a non trivial result in view of the high level of predictability of the present model.

\section{Pati-Salam-like D-brane models}

The effective theory, in the low energy limit, is described by a Pati-Salam gauge group $\mathrm{U}(4) \times \mathrm{Sp}(2)_{L} \times \mathrm{Sp}(2)_{R}$. $\mathrm{U}(4)$ is generated by a stacks of 4 D6-branes and their images $U^{\prime}(4)$ under $\Omega .{ }^{9} \mathrm{Sp}(2)_{L, R}$ are supported on two stacks of two D-branes each lying on top of the $\Omega$-plane. ${ }^{10}$ We also consider three Euclidean $D 2$-branes (or E2-branes) on top of the $\Omega$-plane, corresponding to three Exotic $O(1)$ Instantons. Let us call these $E 2, E 2^{\prime}, E 2^{\prime \prime}$. Quarks and leptons in Left and Right fundamental representations $F_{L, R} \equiv\left(4,2_{L}\right),\left(4^{*}, 2_{R}\right)$, are reproduced as open strings stretching from the U(4)-stack to the Left or Right $\operatorname{Sp}(2)_{L, R^{-}}$ stacks (respectively). Analogously, but at variant w.r.t. the original model [47], Higgs $\bar{H}=$ $\left(4^{*}, 2_{R}\right)$ and its conjugate $H=\left(4,2_{R}\right)$ are introduced as extra intersections of the $\mathrm{U}(4)$ stack with $\operatorname{Sp}(2)_{R}$. Extra color states $\Delta=(10,1,1)$, and their conjugates, are obtained as open strings stretching from the $\mathrm{U}(4)$-stack to its $\Omega$ image $\mathrm{U}(4)^{\prime}$-stack. $\phi_{L L}=(1,3,1)$ and $\phi_{R R}=(3,1,1)$ correspond to strings with both end-points attached to the $\operatorname{Sp}(2)_{L, R}$ (respectively). Higgs fields $h_{L R}=(2,2,1)$ are massless strings stretching from $\operatorname{Sp}(2)_{L}$ to $\operatorname{Sp}(2)_{R}$. The quiver on the left of figure 1 automatically encodes the following superpotential terms [47]:

$$
\begin{aligned}
\mathcal{W}_{Y u k}= & Y^{(0)} h_{L R} F_{L} F_{R}+\frac{Y^{(1)}}{M_{F 1}} F_{L} \phi_{L L} F_{L} \Delta+\frac{Y^{(2)}}{M_{F 2}} F_{R} \phi_{R R} F_{R} \Delta^{c} \\
& +\frac{Y^{(3)}}{M_{F 3}} h_{L R} \phi_{R R} h_{R L} \phi_{L L}+\mu h_{L R} h_{R L}+Y^{(5)} h_{L R} F_{L} \bar{H}+\frac{Y^{(6)}}{M_{F 6}} F_{R} \phi_{R R} \bar{H} \Delta^{c} \\
& +\frac{Y^{(7)}}{M_{F 7}} F_{L} F_{L} F_{R} F_{R}+\frac{Y^{(8)}}{M_{F 8}} F_{L} F_{L} \bar{H} \bar{H}+\frac{Y^{(9)}}{M_{F 9}} F_{L} F_{L} F_{R} \bar{H}
\end{aligned}
$$

\footnotetext{
${ }^{9}$ Let us recall that $\Omega$-planes are introduced for quantum consistency and tadpole cancellations. See references [74-81, 81, 82, 82, 83, 83-86] for a complete discussion of these aspects.

${ }^{10}$ Let us note that, generically, in D-brane models, one cannot construct directly $\mathrm{SU}(N)$ gauge groups. For this reason we cannot obtain directly a PS model, but an extended one, with U(4) rather than SU(4) and $\operatorname{Sp}(2)_{L, R}$ rather than $\mathrm{SU}(2)_{L, R}$. In fact, $N$ parallel branes stacked together (with open strings ending on them) will produce, at low energy limit, $\mathrm{U}(N), \mathrm{SO}(N), \mathrm{Sp}(2 N)$ gauge theories. In particular, $\mathrm{U}(N)$ is obtained if the D-brane stack does not lie on the $\Omega$-plane. On the other hand, if the D-brane stack lies on the $\Omega$-plane, one obtains $\mathrm{SO}(N)$ or $\mathrm{Sp}(2 N)$ (for $\Omega^{\mp}$ respectively). $\Omega$-planes seem necessary in order to produce realistic gauge groups, in which chiral matter can be embedded [87, 88].
} 


$$
\begin{aligned}
\mathcal{W}_{H}= & m_{\Delta} \Delta \Delta^{c}+\frac{1}{4 M_{F 4}}\left(\Delta \Delta^{c}\right)^{2}+\frac{1}{2} m_{L} \phi_{L L}^{2}+\frac{1}{2} m_{R} \phi_{R R}^{2}+\frac{1}{3 !} a_{L} \phi_{L L}^{3} \\
& +\frac{1}{3 !} a_{R} \phi_{R R}^{3}+\mu^{\prime} H \bar{H}+\mu^{\prime \prime} F_{R} H+\frac{Y^{(10)}}{M_{F 10}} \bar{H} \phi_{R R} \bar{H} \Delta^{c} \\
\mathcal{W}_{E 2^{\prime}, E 2^{\prime \prime}}= & \frac{Y^{\prime(1)}}{\mathcal{M}_{0}^{\prime}} \epsilon^{i j k l} \epsilon^{i^{\prime} j^{\prime} k^{\prime} l^{\prime}} \Delta_{i i^{\prime}}^{c} \Delta_{j j^{\prime}}^{c} \Delta_{k k^{\prime}}^{c} \Delta_{l l^{\prime}}^{c}+\frac{Y^{\prime \prime}(1)}{\mathcal{M}_{0}^{\prime \prime}} \epsilon^{i j k l} \epsilon^{i^{\prime} j^{\prime} k^{\prime} l^{\prime}} \Delta_{i i^{\prime}} \Delta_{j j^{\prime}} \Delta_{k k^{\prime}} \Delta_{l l^{\prime}}
\end{aligned}
$$

$Y^{(\ldots)}$ are $3 \times 3$ Yukawa matrices; the mass scales $M_{F \ldots}$ are considered as free parameters: they depend on the particular completion of our model, i.e. they could be near $M_{S}$, the string scale, as well as at lower scales. ${ }^{11}$ The super-potential terms (2.3) can be generated by two E2-brane instantons shown in figure 1: $O(1)^{\prime}, O(1)^{\prime \prime}$ intersect twice the U(4) stack and $O(1)$ intersects twice the $\mathrm{U}(4)$-stack and once the $\operatorname{Sp}(2)_{R}$-stack $\left(2_{R}\right.$ on the left side of figure 1). In fact, fermionic modulini $\tau_{i}, \tau_{i}^{\prime}, \omega_{\alpha}^{\prime}$ appear as massless excitations of open strings ending on $\mathrm{U}(4)-O(1), \mathrm{U}(4)-O(1)^{\prime}, \mathrm{Sp}(2)_{R}-O(1)^{\prime}$ respectively; $i=1,4$ and $\alpha=$ 1,2 are indices of $\mathrm{U}(4)$ and $\mathrm{Sp}(2)_{R}$ respectively. Integrating over the fermionic modulini, we exactly recover the interactions $(2.5)$ and (2.3), as shown in [47] or in [89-91, 93] in different contexts. ${ }^{12}$ The dynamical scales generated in (2.3) are $\mathcal{M}_{0}^{\prime}=Y^{\prime(1)} M_{S} e^{+S_{E 2^{\prime}}}$ and $\mathcal{M}_{0}^{\prime \prime}=Y^{\prime \prime}(1) M_{S} e^{+S_{E 2^{\prime \prime}}}$, where $S_{E 2^{\prime}, E 2^{\prime \prime}}$ depend on geometric moduli, associated to 3-cycles of the $C Y_{3}$, around which $E 2^{\prime}, E 2^{\prime \prime}$ are wrapped.

The spontaneous breaking pattern down to the (MS)SM (minimal supersymmetric standard model) is

$$
\begin{aligned}
\mathrm{U}(4) \times \mathrm{Sp}(2)_{L} \times \mathrm{Sp}(2)_{R} \underset{\langle\mathrm{Stu}\rangle}{\longrightarrow} \mathrm{SU}(4) \times \mathrm{Sp}(2)_{L} \times \mathrm{Sp}(2)_{R} \\
\left\langle\overrightarrow{H, H, h\rangle} \mathrm{SU}(3) \times \mathrm{Sp}(2)_{L} \times \mathrm{U}(1)_{Y}\right.
\end{aligned}
$$

(Stu stands for Stückelberg, see below) and $h_{L R}$ contain the standard Higgses for the final electroweak symmetry breaking. Decuplets decompose as $\Delta^{c}=\Delta_{6}^{c}+T^{c}+S^{c}$, with $\Delta_{6}=6_{Y=+2 / 3}, T=3_{Y=-2 / 3}, S=1_{Y=-2}$, and the singlet $S$ takes a VEV.

Let us note that the extra $\mathrm{U}(1)_{4} \subset \mathrm{U}(4)_{c}$ is anomalous in gauge theory. In string theory a generalization of the Green-Schwarz mechanism can cure these anomalies. Generalized Chern-Simons (GCS) terms are generally required in this mechanism. The new vector boson $Z^{\prime}$ associated to $\mathrm{U}(1)_{4}$ gets a mass via a Stückelberg mechanism. ${ }^{13}$

\footnotetext{
${ }^{11}$ The mass terms $m_{\Delta}$ and $m_{L, R}$ can be generated by R-R or NS-NS 3-forms fluxes in the bulk, in a T-dual Type IIB description, i.e. $m_{\Delta} \sim \Gamma^{i j k}\left\langle\tau H_{i j k}+i F_{i j k}\right\rangle, m_{L, R} \sim \Gamma^{i j k}\left\langle\tau H_{i j k}^{(L, R)}+i F_{i j k}^{(L, R)}\right\rangle$, with $H_{3}$ RR-RR and $F_{3}$ NS-NS 3-forms. In general, $H_{3}, F_{3}$ are not flavour diagonal since fluxes through different cycles, wrapped by different D-branes, could be different. For recent discussions of mass deformed quivers and dimers see [94].

${ }^{12}$ In [89-91, 93] Majorana masses for neutrinos are completely generated by exotic instantons.

${ }^{13}$ See [95-106] for discussions about these aspects in different contexts, and [47] for comments on implications in PS models, like $Z_{R}-Z^{\prime}$ mixings or GCS interactions $Z_{R}-Z^{\prime}-Z$ or $Z_{R}-Z^{\prime}-\gamma$ etc (where $Z_{R}$ is the $\mathrm{SU}(2)_{R} Z$-boson). Another implementation of the Stückelberg mechanism is in the realization of Lorentz Violating Massive gravity [107-109]. Recently, geodetic instabilities of Stückelberg Lorentz Violating Massive gravity were discussed in [110] (and also connected to solutions of naked singularities discussed in [111]). We would like to stress that GCS terms generate UV divergent triangles that are cured by considering UV completions with KK states or string excitations. For issues in scattering amplitudes and collider physics see [112]. See also $[113,114]$ for a string-inspired non-local field model of string theory.
} 

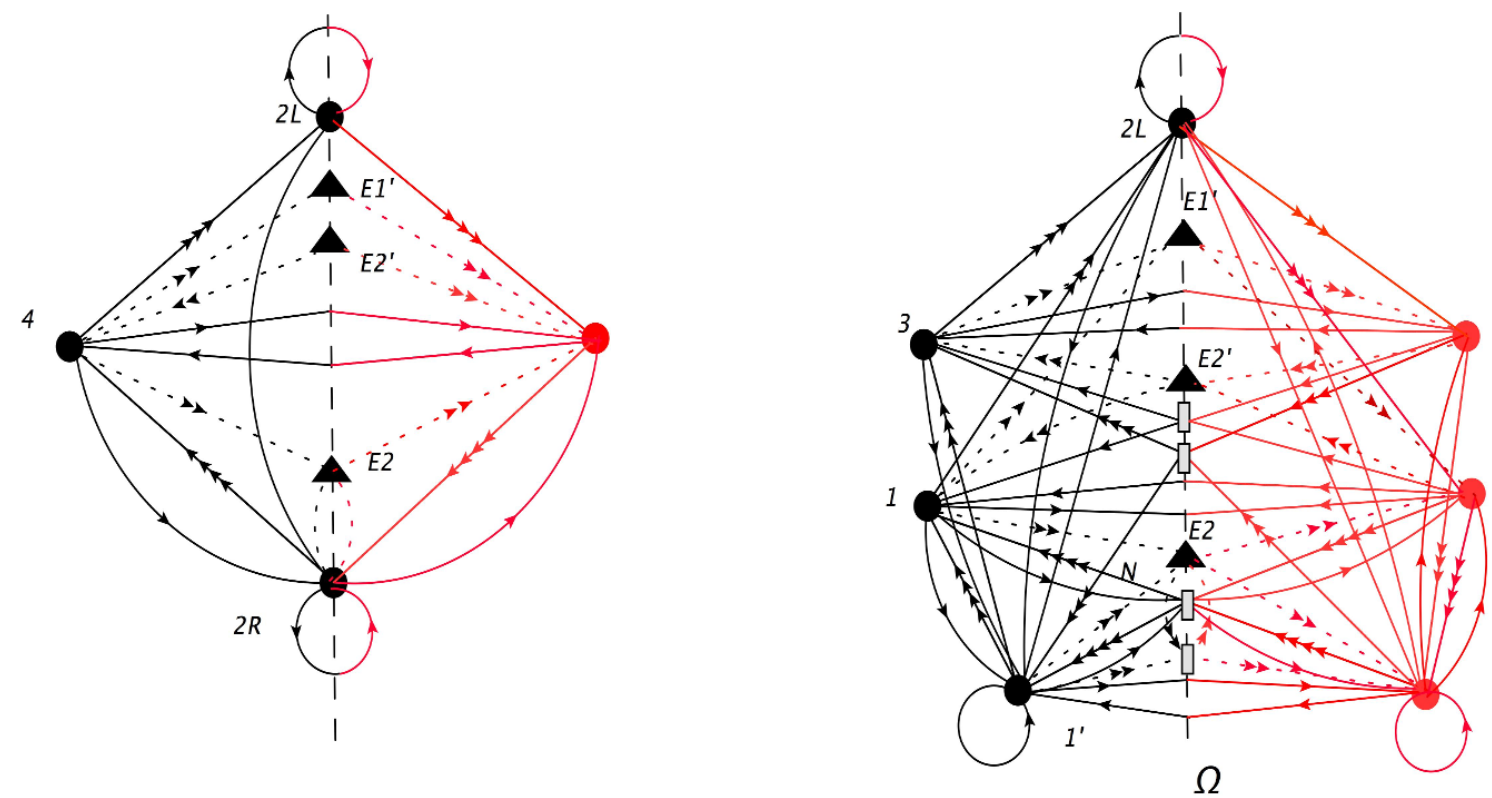

Figure 1. On the left, the unoriented quiver for a Pati-Salam-like model $\mathrm{U}(4) \times \operatorname{Sp}(2)_{L} \times \operatorname{Sp}(2)_{R}$ is shown. Circles, labeled by $4,2_{L}, 2_{R}$, correspond to the $\mathrm{U}(4), \operatorname{Sp}(2)_{L}, \operatorname{Sp}(2)_{R}$ gauge groups, respectively. The $\mathrm{U}(4)$ stack is identified with its mirror image through an $\Omega^{+}$-plane. $\operatorname{Sp}(2)_{L, R}$ correspond to stacks of two D6-branes lying on the $\Omega^{+}$-plane. The triangles are $E 2$-branes lying on the $\Omega^{+}$ plane, corresponding to $O(1)$ instantons. $E 2^{\prime}, E 2^{\prime \prime}$-instantons generate a quartic superpotential for $\Delta(10,1,1)$ and $\Delta^{c}(10,1,1)$, leading to an effective Majorana mass for the neutron. On the right, the effective unoriented quiver theory after Higgsing via $H, \bar{H}$ is shown. From the quiver on the left to the one on the right, extra undesired modulini appear, that are assumed to be lifted by a combination of higgsing and fluxes. The E2-instanton generates a PMNS mass matrix for neutrinos. The PS-like quiver generates the (MS)SM-like quiver on the right side after splitting the $\operatorname{Sp}(2)_{R}$ D-branes from the $\Omega^{+}$-plane.

The final effective (MS)SM embedding quiver that we will consider is obtained from the previous SUSY PS-like quiver through a splitting of nodes $4 \rightarrow 3+1$ and $2_{R} \rightarrow 1+1^{\prime}$. In this new quiver, $E 2$ intersects $\mathrm{U}(1)$ and $\hat{U}(1)^{\prime}$ as shown on the right of figure 1, where $\hat{U}^{\prime}(1)$ is the $\Omega$-image of $U^{\prime}(1)$. In the Higgsing from SUSY PS-like quiver to SUSY SMlike, extra undesired modulini are obtained. In particular, colored modulini at E2-U(3) intersections. We assume that these modulini are lifted out by Higgsings and fluxes. This technical aspect deserves future investigation beyond the purposes of this paper. As a consequence, an extra mass matrix term is non-perturbatively generated

$$
\mathcal{W}_{E 2}=\frac{1}{2} \mathcal{M}_{a b}^{\prime} N_{R}^{a} N_{R}^{b}
$$

where $N_{R}^{a}$ are RH neutrinos ( $a=1,2,3$ label neutrino species), contained, as singlet,

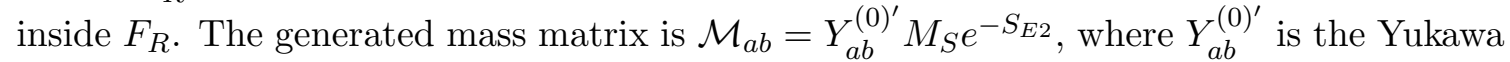
matrix parameterizing masses and mixings among $\mathrm{RH}$ neutrinos, depending of course on the particular E2 intersections with ordinary D6-branes stacks. Let us note that the superpotential (2.5) can be generated only after spontaneous symmetry breaking of $\mathrm{U}(4)_{c}$ 
down to $\mathrm{U}(3)_{c}$, and $\operatorname{Sp}(2)_{R}$ down to $U^{\prime}(1)$. This will impose bounds on the parameters that we will discuss in section 2 .

Now, let us discuss electroweak symmetry breaking in our present model: as mentioned before, this is due to the VEVs $\left\langle h_{L R}\right\rangle$ of the complex Higgs bi-doublets $h_{L R}$ yielding the tree-level mass relations for leptons and quarks

$$
m_{d}=m_{e} \quad \text { and } \quad m_{u}=m_{D}
$$

where $m_{D}$ are Dirac masses of neutrinos. From (2.6), tight hierarchy constraints on RH neutrino masses are predicted: as a result the neutrino's hierarchy is related to the up-quarks. It is interesting to observe that the hierarchy obtained at the perturbative level (with closedstring fluxes generating the $M_{2}$ scale) is corrected by exotic instantons, parametrized by $\mathcal{M}_{a b}$. Left-Right symmetry breaking pattern implies

$$
m_{D}=m_{u} \quad \text { and } \quad V_{L}=V_{\mathrm{CKM}}
$$

with $V_{\mathrm{CKM}}$ the Cabibbo-Kobayashi-Maskawa matrix. We obtain the mass matrix

$$
M=\left(\begin{array}{cc}
0 & m_{D} \\
m_{D} & M_{R}
\end{array}\right)
$$

In our case, RH neutrino masses are

$$
M_{R}=M_{R}^{P}+M_{R}^{E 2^{\prime}}
$$

where

$$
M_{R}^{P}=\left\langle\phi_{R R}\right\rangle\left\langle S^{c}\right\rangle / M_{2}
$$

and

$$
M_{R}^{E 2}=\mathcal{M}_{a b}^{\prime}
$$

as shown in [47].

From the usual see-saw formula one obtains the light neutrino mass matrix $m_{\nu}$

$$
m_{\nu} \simeq-m_{D}\left(M_{R}^{P}+M_{R}^{E 2}\right)^{-1} m_{D}
$$

A natural situation for our quiver is that $E 2^{\prime}$ induce non-perturbative mass terms for $\mathrm{RH}$ neutrinos of the same order, i.e. $M_{R, 1}^{E 2} \simeq M_{R, 2}^{E 2} \simeq M_{R, 3}^{E 2}$ where 1,2,3 are generation indices. As a consequence, $M_{R, 1,2,3}^{E 2} \simeq 10^{9 \div 13} \mathrm{GeV}$ and we obtain a highly degenerate $\mathrm{RH}$ mass spectrum in a good range for leptogenesis, non-perturbative mass corrections are higher than or at least of the same order as the perturbative ones. Naturally, such a situation does not imply a highly degenerate LH mass spectrum, since a large quark-lepton hierarchy remains encoded in $m_{D}$. The see-saw formula can be inverted as

$$
M_{R}=M_{R}^{p}+M_{R}^{E 2} \simeq-m_{D} m_{\nu}^{-1} m_{D}
$$

since in our model $m_{D}=m_{D}^{T}$. From (2.10) one can get information on the RH neutrino mass matrix $M_{R}$ by using data on LH neutrino mass matrix $m_{\nu}$, and assuming a 
quark-lepton symmetry. In general, a quark-lepton symmetry complicates BAU mechanisms because it imposes a strong hierarchy in the neutrino sector: under the assumption that $v_{1} v_{2} / M_{2} \simeq 10^{11 \div 13} \mathrm{GeV}$ with $v_{1}=\left\langle\phi_{R R}\right\rangle$ and $v_{2}=\left\langle\Delta^{c}\right\rangle$, the lightest RH eigenstate $N_{1}$ takes a mass much smaller than the Davidson-Ibarra bound [27], $M_{N_{1}} \ll 10^{9} \mathrm{GeV}$, i.e. $N_{1}$ decays cannot guarantee a sufficient production of lepton asymmetry. Fortunately, non perturbative $E 2$ contributions can generate a compact $\mathrm{RH}$ neutrino spectrum above the DI bound, i.e. the mass eigenvalues of $\mathrm{RH}$ neutrino mass matrix are highly degenerate and higher than $10^{9} \mathrm{GeV}$. We would like to stress that, unlike $\mathrm{SO}(10)$ GUTs, our model provides a natural mechanism to obtain a compact $\mathrm{RH}$ neutrino hierarchy. Let us also observe that, after the splitting in figure 1, we obtain an effective cubic interaction term $\left(\left\langle S^{c}\right\rangle / \mathcal{M}_{0}\right) \epsilon_{i j k}^{\mathrm{SU}(3)} \epsilon_{i^{\prime} j^{\prime} k^{\prime}}^{\mathrm{SU}(3)} \Delta_{6}^{c^{i i^{\prime}}} \Delta_{6}^{c^{j j^{\prime}}} \Delta_{6}^{c^{k k^{\prime}}}$ which violates Baryon number as $\Delta B=2$ and generates a Majorana mass for neutrons [47], as mentioned in the introduction. On the other hand, exotic instantons can preserve discrete sub-symmetries $Z_{2}^{(\Delta B, \Delta L=1)}$, avoiding proton destabilization, but allowing $\Delta L, \Delta B= \pm 2$ processes. However, $\Delta B=2$ violating operators can also destabilize the proton if one consider all $\Delta L=1$ mixing terms among $F_{L, R}$ and $H, \bar{H}$ in (2.1). Higher order operators of this kind are generated by fluxes, so that one can naturally assume that they are suppressed by a mass scale larger than $M_{S}$.

So, potentially dangerous terms are

$$
\mathcal{W}_{Y^{(5)}, \Delta L=1}=Y^{(5)} h_{L R} F_{L}\langle\bar{H}\rangle \quad \text { and } \quad \mathcal{W}_{\mu, \Delta L=1}=\mu^{\prime \prime} F_{R} H
$$

These terms are easily understood: $\bar{H}$ is like a fourth generation of $F_{R}$. So that, calling $\mathcal{F}_{R}^{f=1,4}=\left(F_{R}^{f=1,3}, \bar{H}\right)$, they generically mix through

$$
\mathcal{W}_{\mu_{f}}=\mu_{f} H \mathcal{F}_{R}^{f}=\mu^{\prime} H \bar{H}+\mu^{\prime \prime} H F_{R}
$$

Such mass terms can be diagonalized so that the mixing term $H F_{R}$ can be rotated away in the mass eigenstate basis. Similarly, $\mathcal{W}_{Y^{(5)}}$ can be incorporated in the standard Yukawa term as

$$
\mathcal{W}_{\mathcal{Y}^{f}}=\mathcal{Y}_{f^{\prime}=1,3 ; f=1,4} h_{L R} F_{L}^{f^{\prime}} \mathcal{F}_{R}^{f}=h_{L R}\left[Y_{f^{\prime}=1,3, f=1,3}^{(0)} F_{L}^{f^{\prime}} F_{R}^{f}+Y_{f^{\prime}=1,3}^{(5)} F_{L}^{f^{\prime}} \bar{H}\right]
$$

In order to avoid proton destabilization, we can impose the following condition on matrices $\mu_{f}$ and $\mathcal{Y}^{f}$

$$
\mu_{f} \mathcal{Y}^{f}=0
$$

Relation (2.11) automatically guarantees matrices of the form

$$
\begin{aligned}
& \mu_{f}=\left(\mu^{\prime}, 0,0,0\right)^{T} \\
& \mathcal{Y}_{f}=\left(0, Y_{f=1}^{(0)}, Y_{f=2}^{(0)}, Y_{f=3}^{(0)}\right)
\end{aligned}
$$

in the basis $\mathcal{F}_{R}^{f}=\left(F_{R}^{f=1,2,3}, \bar{H}\right)$.

A natural geometric explanation of eq. (2.11) could come from global intersecting Dbrane models, consistently completing our local one in the Calabi-Yau singularity. The quiver in figure 1 apparently seems to democratically consider different flavors, like $\mathcal{F}_{R}^{f}$. 
However, the presence of internal bulk R-R or NS-NS fluxes can discriminate different intersections of two stacks of D6-branes i.e. different flavors from one another. Alternatively, one can consider that the local quiver theory in figure 1 could come from a (or more) Gepner model(s). In Gepner models, the Calabi-Yau space has a more complicated geometry than for example a toroidal orbifold, inducing accidental discrete symmetries in the low energy limits. For example, the intersections of two stacks on a torus are geometrically equivalent, while in a complicated topological deformation of a torus "flavor democracy" is broken. This affects the vertex operators of an open string massless fermion $\mathcal{V}_{F}=\mathcal{V}_{S} \Sigma_{f}^{\text {int }}$, where $\mathcal{V}_{S}=u^{\alpha}(k) S_{\alpha} e^{-\phi / 2} e^{i k X}$ accounts for the space-time part, while $\Sigma_{f}^{\text {int }}$ is an internal spin field depending on the flavor. Similarly for massless scalars $\mathcal{V}_{B}=\Psi_{f}^{\text {int }} e^{-\phi} e^{i k X}$, with $\Psi_{f}^{\text {int }}$ being a chiral primary operator. A Yukawa coupling, like $h_{L R} F_{L} \mathcal{F}_{R}$, will give rise to a flavor matrix $Y_{f_{1} f_{2} f_{3}}$ proportional to $\left\langle\Psi_{f_{1}}^{\text {int }} \Sigma_{f_{2}}^{\text {int }} \Sigma_{f_{3}}^{\text {int }}\right\rangle$. As a consequence, the suppression of $\mathcal{W}_{Y^{(5)}, \Delta L=1}$ can be geometrically understood as emerging from different inequivalent intersections among the same stacks of branes. ${ }^{14}$

\subsection{Free parameters}

In this section we will comment on the relevant parameters in our model and clarify our assumptions.

\subsubsection{Supersymmetry and string scale}

First, let us clarify the role of supersymmetry in our considerations. Clearly, if the SUSY breaking scale is assumed to be $M_{\mathrm{SUSY}} \simeq 1 \mathrm{TeV}$, this will introduce several extra parameters relevant for leptogenesis. A TeV-scale SUSY will complicate one-loop (n-loops) contributions, introducing extra CP-violating phases in RH-neutrino decays. Here, we will assume that supersymmetry has nothing to do with the hierarchy problem of the Higgs mass, i.e. SUSY has the role to stabilize instanton calculations and to eliminate tachyonic states from the present string model. While the second aspect is crucial for the consistency of our model, saving us from "fighting" with instabilities, and imposing a bound on the SUSYscale as $M_{\mathrm{SUSY}} \simeq M_{S}$, the first aspect is "less fundamental", since it only has the role of simplifying istanton calculations. This requires $M_{\mathrm{SUSY}} \simeq M_{S} e^{-S_{E 2}} \gtrsim 10^{9} \mathrm{GeV}$. As a result, supersymmetric particles do not give any relevant contributions to $\mathrm{RH}$ neutrino decays. ${ }^{15}$

\subsubsection{Relevant effective Lagrangian and free parameters}

After the spontaneous breaking of SUSY, U(4) symmetry and Left-Right symmetry, the effective Lagrangian in the neutrino sector reads

$$
\mathcal{L}_{\text {eff }}^{\nu}=Y^{(0)}\left\langle h_{u}\right\rangle l \nu_{R}+\frac{Y^{(2)}}{M_{2}} \nu_{R}\left\langle\varphi_{R R}\right\rangle \nu_{R}\left\langle\delta^{c}\right\rangle+Y^{(0)^{\prime}} M_{S} e^{-S_{E 2}} \nu_{R} \nu_{R}
$$

\footnotetext{
${ }^{14}$ For recent literature on emergent discrete symmetries in (MS)SM-like and PS-like models, see [115, 139] and references therein.

${ }^{15}$ One could speculate that dark matter is a hidden parallel system of intersecting D-branes. Implications in direct detection of such a scenario was studied in [148].
} 
where $h_{u}$ is the scalar component of the superfield $H_{u}$ contained in the bi-doublet superfield $h_{L R}, \nu_{R}$ are the $\mathrm{RH}$ neutrinos, the fermionic component of the the $\mathrm{RH}$ neutrino supermultiplets, $\varphi_{R R}, \delta^{c}$ are the scalar components of the supermultiplets $\phi_{R R}, \Delta^{c}$.

Therefore, the number of relevant free parameters in the neutrino sector is

$$
N_{\text {f.p. }}=n_{Y 0}+n_{Y 2}+n_{Y 0^{\prime}}+n_{\mathrm{VEV} 1}+n_{\mathrm{VEV} 2}+n_{\text {Flux }}+n_{E 2}=22
$$

(f.p. stands for free-parameters) where $n_{Y 0,2}=6$ are the number of free parameters in the Yukawa matrices $Y^{(0)}, Y^{(2)}, Y^{(0)^{\prime}}$ respectively; $n_{\mathrm{VEV} 1, \mathrm{VEV} 2}$ account for the number of ratios between extra VEVs $v_{1,2}$ with respect to $v_{\mathrm{EW}}$, i.e. $z_{1}=v_{1} / v_{\mathrm{EW}}$ and $z_{2}=v_{2} / v_{\mathrm{EW}}$; $n_{\text {Flux }}=1$ is the number of non-perturbative scales generated by fluxes entering in the neutrino sector, i.e. $M_{F 2}$ (or $z_{3}=M_{F 2} / v_{\mathrm{EW}}$ ); $n_{E 2}$ parameterizes the size of the 3 -cycle wrapped by E2-brane.

Under reasonable assumptions, the number of free parameters can be significantly reduced. In the following analysis, we will suppose a dominance of non-perturbative effects: $M_{R}^{E 2} \gg M_{R}^{P}$ (all matrix parameters). In this case, $n_{\mathrm{VEV} 1, \mathrm{VEV} 2 \text {,Flux,Y2 }}$ are irrelevant, as they are related to tiny extra corrections. In this case, the mass matrix of $\mathrm{RH}$ neutrinos is practically completely generated by the $E 2$-instanton! AB: the hierarchy $M_{R}^{E 2} \gg M_{R}^{P}$ can be understood as follows. The E2-instanton generates a mass matrix for neutrinos with an absolute value $M_{S} e^{-\Pi_{3} / g_{s}}$, where $\Pi_{3}$ is the volume of 3-cycles wrapped by the $E 2$-instanton on $C Y_{3}$. Volumes of 3 -cycles (in string units) can be as small as $\Pi_{3} \simeq 1$, or as large as $\Pi_{3} \gg 1$. In other words, the hierarchy among $\mathrm{RH}$ neutrino masses and the string scale can be considered as a free parameter. On the other hand, the $Y^{(2)}$-term is suppressed by the scale of the non-perturbative flux, that can easily be near the string-scale so as to justify the assumed hierarchy $M_{R}^{E 2} \gg M_{R}^{P}$.

As a consequence, the number of relevant parameters will simply be

$$
N_{\text {f.p. }} \simeq n_{Y 0}+n_{Y 0^{\prime}}+n_{E 2}=6+6+1=13
$$

Let us note that such a situation requires $v_{1} v_{2} / M_{F 2} \ll 10^{9} \mathrm{GeV}$. But $v_{1,2}<v_{R}$ with $v_{R} \gtrsim 10^{9} \mathrm{GeV}$ : exotic instanton effects are related to a Stückelberg mechanism for $\mathrm{U}(1)_{B-L}$,

otherwise they will violate the B-L gauge symmetry. On the other hand, $v_{R} \gtrsim 10^{9} \mathrm{GeV}$ since exotic instantons have to distinguish $\mathrm{RH}$ neutrinos from $E^{c}$ at this very scale! As a consequence, $M_{F 2} \gg 10^{9} \mathrm{GeV}$ satisfies these bounds. This situation seems natural: $M_{F 2}$ are related to closed-string fluxes, i.e. another kind of quantum gravity effects.

\section{Phenomenology in neutrino physics}

In this section we derive our predictions for yet-unknown low energy neutrino parameters, the mass of the lowest neutrino state and the phases of the PMNS (Pontecorvo-MakiNakagawa-Sakata) matrix.

\subsection{Conditions for a compact RH neutrino spectrum}

As mentioned in section 2, the Dirac neutrino mass matrix $m_{D}$ is symmetric, thus it can be diagonalized by a single unitary matrix $V_{L}[140-142]$

$$
m_{D}=V_{L}^{\dagger} m_{D}^{\operatorname{diag}} V_{L}^{*}
$$


where $m_{D}^{\operatorname{diag}} \equiv \operatorname{diag}\left(m_{D 1}, m_{D 2}, m_{D 3}\right)$ with real and non-negative eigenvalues $m_{(D 1, D 2, D 3)}$. The seesaw condition expressed in eq. (2.10) yields

$$
M_{R}=-V_{L}^{\dagger} m_{D}^{\operatorname{diag}} A m_{D}^{\operatorname{diag}} V_{L}^{*}
$$

where we have defined a matrix $A$, symmetric by construction, as

$$
A=V_{L}^{*} m_{\nu}^{-1} V_{L}^{\dagger}
$$

In terms of the matrix elements of $A$ and $V_{L}$, the RH mass matrix elements become

$$
\begin{aligned}
M_{R 11}= & -A_{11} V_{L 11}^{* 2} m_{D 1}^{2}-A_{22} V_{L 21}^{* 2} m_{D 2}^{2}-A_{33} V_{L 31}^{* 2} m_{D 3}^{2}+ \\
& -2 A_{12} V_{L 11}^{*} V_{L 21}^{*} m_{D 1} m_{D 2}-2 A_{13} V_{L 11}^{*} V_{L 31}^{*} m_{D 1} m_{D 3}-2 A_{23} V_{L 11}^{*} V_{L 21}^{*} m_{D 2} m_{D 3} \\
M_{R 12}= & -A_{11} V_{L 11}^{*} V_{L 12}^{*} m_{D 1}^{2}-A_{22} V_{L 21}^{*} V_{L 22}^{*} m_{D 2}^{2}-A_{33} V_{L 31}^{*} V_{L 32}^{*} m_{D 3}^{2}+ \\
& -A_{12}\left(V_{L 12}^{*} V_{L 21}^{*}+V_{L 11}^{*} V_{L 22}^{*}\right) m_{D 1} m_{D 2}-A_{13}\left(V_{L 12}^{*} V_{L 31}^{*}+V_{L 11}^{*} V_{L 32}^{*}\right) m_{D 1} m_{D 3}+ \\
& -A_{23}\left(V_{L 22}^{*} V_{L 31}^{*}+V_{L 21}^{*} V_{L 32}^{*}\right) m_{D 2} m_{D 3} \\
M_{R 13}= & -A_{11} V_{L 11}^{*} V_{L 13}^{*} m_{D 1}^{2}-A_{22} V_{L 21}^{*} V_{L 23}^{*} m_{D 2}^{2}-A_{33} V_{L 31}^{*} V_{L 33}^{*} m_{D 3}^{2}+ \\
& -A_{12}\left(V_{L 13}^{*} V_{L 21}^{*}+V_{L 11}^{*} V_{L 23}^{*}\right) m_{D 1} m_{D 2}-A_{13}\left(V_{L 13}^{*} V_{L 31}^{*}+V_{L 11}^{*} V_{L 33}^{*}\right) m_{D 1} m_{D 3}+ \\
& -A_{23}\left(V_{L 23}^{*} V_{L 31}^{*}+V_{L 21}^{*} V_{L 33}^{*}\right) m_{D 2} m_{D 3} \\
M_{R 22}= & -A_{11} V_{L 12}^{* 2} m_{D 1}^{2}-A_{22} V_{L 22}^{* 2} m_{D 2}^{2}-A_{33} V_{L 32}^{* 2} m_{D 3}^{2}+ \\
& -2 A_{12} V_{L 12}^{*} V_{L 22}^{*} m_{D 1} m_{D 2}-2 A_{13} V_{L 12}^{*} V_{L 32}^{*} m_{D 1} m_{D 3}-2 A_{23} V_{L 22}^{*} V_{L 32}^{*} m_{D 2} m_{D 3} \\
M_{R 23}= & -A_{11} V_{L 12}^{*} V_{L 13}^{*} m_{D 1}^{2}-A_{22} V_{L 22}^{*} V_{L 23}^{*} m_{D 2}^{2}-A_{33} V_{L 32}^{*} V_{L 33}^{*} m_{D 3}^{2}+ \\
& -A_{12}\left(V_{L 13}^{*} V_{L 22}^{*}+V_{L 12}^{*} V_{L 23}^{*}\right) m_{D 1} m_{D 2}-A_{13}\left(V_{L 13}^{*} V_{L 32}^{*}+V_{L 12}^{*} V_{L 33}^{*}\right) m_{D 1} m_{D 3}+ \\
& -A_{23}\left(V_{L 23}^{*} V_{L 32}^{*}+V_{L 22}^{*} V_{L 33}^{*}\right) m_{D 2} m_{D 3} \\
M_{R 33}= & -A_{11} V_{L 13}^{*} m_{D 1}^{2}-A_{22} V_{L 23}^{* 2} m_{D 2}^{2}-A_{33} V_{L 33}^{* 2} m_{D 3}^{2}-2 A_{12} V_{L 13}^{*} V_{L 23}^{*} m_{D 1} m_{D 2}+ \\
& -2 A_{13} V_{L 13}^{*} V_{L 33}^{*} m_{D 1} m_{D 3}-2 A_{23} V_{L 23}^{*} V_{L 33}^{*} m_{D 2} m_{D 3}
\end{aligned}
$$

Since the matrix $M_{R}$ is also symmetric by construction, one has $M_{R i j}=M_{R j i}$ for any $i, j=1,2,3$. Motivated by quark-lepton symmetry, we assume, as for quarks, a large hierarchy in the eigenvalues of the Dirac mass matrix for leptons, that is

$$
m_{D 1} \ll m_{D 2} \ll m_{D 3}
$$

The hierarchy assumption in (3.5) implies that the elements of $A$ are at most mildly hierarchical, and the same holds for the RH neutrino spectrum. Therefore only specific constraints on the $A$ matrix can enforce the conditions that ensure that the $\mathrm{RH}$ neutrino spectrum is compact. We can immediately see that a generically compact $\mathrm{RH}$ spectrum would result by suppressing the entries proportional to $A_{23}$ and $A_{33}$. In that case, all matrix elements become of the same order of magnitude, that is $m_{D 1} m_{D 3} \sim m_{D 2}^{2}$. In first approximation, we can set

$$
A_{23}=A_{33}=0 .
$$


Let us stress that while the approximation (3.6) has the virtue of simplifying the analysis, a generic compact $\mathrm{RH}$ neutrino spectrum can be obtained by fixing the $A_{23}$ and $A_{33}$ values to any sufficiently small number.

The precise form of the $V_{L}$ matrix is not crucial to ensure the compactness of the $\mathrm{RH}$ spectrum, provided it does not have unnaturally large matrix elements. Guided by the symmetries of the model, discussed in section 2, we assume that in the diagonal basis for the down-quarks and charged leptons mass matrices, the unitary rotation $V_{L}$ that diagonalizes the symmetric matrix $m_{D}$ coincides with the Cabibbo-Kobayashi-Maskawa (CKM) matrix that diagonalizes $m_{u}$. In other terms, we set, according to eq. (2.7)

$$
V_{L}=V_{\mathrm{CKM}}
$$

where $V_{\mathrm{CKM}}$ is the CKM matrix encoding quark mixing.

\subsection{Low energy observables}

The PMNS matrix is the lepton conterpart of the CKM mixing matrix in the quark sector. If neutrinos are Majorana particles, there are two more physical phases with respect to the CKM matrix. By adopting the standard parametrization in terms of three Euler mixing angles $\theta_{12}, \theta_{23}$ and $\theta_{13}$, a Dirac phase $\delta$, and two Majorana phases $\alpha$ and $\beta$, the PMNS mixing matrix can be written as:

$$
U_{\mathrm{PMNS}}=U_{\mathrm{PMNS}}^{\prime}\left(\theta_{12}, \theta_{23}, \theta_{13}, \delta\right) \times \operatorname{diag}\left(1, e^{i \alpha}, e^{i \beta}\right) .
$$

where

$$
U_{\mathrm{PMNS}}^{\prime}=\left(\begin{array}{ccc}
c_{12} c_{13} & s_{12} c_{13} & s_{13} e^{-i \delta} \\
-s_{12} c_{23}-c_{12} s_{23} s_{13} e^{i \delta} & c_{12} c_{23}-s_{12} s_{23} s_{13} e^{i \delta} & s_{23} c_{13} \\
s_{12} s_{23}-c_{12} c_{23} s_{13} e^{i \delta} & -c_{12} s_{23}-s_{12} c_{23} s_{13} e^{i \delta} & c_{23} c_{13}
\end{array}\right)
$$

Here $c_{i j}=\cos \theta_{i j}$ and $s_{i j}=\sin \theta_{i j}$, with $i$ and $j$ labeling families that are coupled through that angle $(i, j=1,2,3)$. In the basis in which the charged lepton mass matrix is diagonal, $U_{\text {PMNS }}$ diagonalizes the effective neutrino mass matrix

$$
m_{\nu}=U_{\mathrm{PMNS}}^{*} m_{\nu}^{\operatorname{diag}} U_{\mathrm{PMNS}}^{\dagger}
$$

where

$$
m_{\nu}^{\operatorname{diag}}=\operatorname{diag}\left(m_{1}, m_{2}, m_{3}\right)
$$

Since the matrix $V_{L}$ is also unitary, we choose the same parameterization as for the PMNS matrix, eq. (3.9), distinguishing the $V_{L}$ parameters with a prime superscript: $s_{12}^{\prime}, s_{23}^{\prime}$, $s_{13}^{\prime}, \delta^{\prime}$. Their values are the same as the ones in the CKM matrix because of the assumption $V_{L}=V_{\mathrm{CKM}}$, discussed in section 3.1.

In section 2.1.2 we have operated a counting of the fundamental free parameters of the model, and found 13 real parameters in the case of dominance of non-perturbative effects. Under the assumption of symmetry expressed by eq. (2.7), the values of these 13 real parameters are constrained by observables in the up-type quark and neutrino sectors. 
They are: the three quark masses $m_{u}, m_{c}, m_{t}$, the two neutrino mass-squared differences $\Delta m_{21}^{2}, \Delta m_{32}^{2}$, the three CKM mixing angles $\theta_{12}^{\prime}, \theta_{23}^{\prime}, \theta_{13}^{\prime}$ and the three PMNS mixing angles $\theta_{12}, \theta_{23}, \theta_{13}$, amounting to 11 real observables. Imposing on the complex elements of the matrix $A$ the two additional conditions in (3.6), $A_{23}=A_{33}=0$, implies that the set of real fundamental parameters must satisfy two additional requirements, that is $\operatorname{Re}\left(A_{23}\right)=$ $\operatorname{Re}\left(A_{33}\right)=0$. Thus the parameter space of the model remains completely determined, allowing to obtain a quantitative prediction for the absolute neutrino mass scale $m_{1}$.

The matrix $A$ can be expressed in terms of the observables $V_{L}, U_{\mathrm{PMNS}}$ and $m_{\nu}^{\text {diag }}$ as

$$
A=\left(V_{L} U_{\mathrm{PMNS}}^{*}\right)^{*} \frac{1}{m_{\nu}^{\text {diag }}}\left(V_{L} U_{\mathrm{PMNS}}^{*}\right)^{\dagger} .
$$

This equality connects $A$ to the observables listed before, and the conditions $A_{23}=A_{33}=0$ determine two relations among them, that we generically indicate with

$$
\begin{aligned}
& f\left(\left[\theta_{i j}^{\prime}, \delta^{\prime}, \theta_{12}, \theta_{23}, \theta_{13}, \Delta m_{21}^{2}\right] ; \delta, m_{1}, \alpha, \beta\right)=0 \\
& g\left(\left[\theta_{i j}^{\prime}, \delta^{\prime}, \theta_{12}, \theta_{23}, \theta_{13}, \Delta m_{31}^{2}\right] ; \delta, m_{1}, \alpha, \beta\right)=0
\end{aligned}
$$

where $f$ and $g$ are known functions. We have eliminated $m_{2}$ and $m_{3}$ by using their relations with their mass-squared differences, $m_{2}^{2}=m_{1}^{2}+\Delta m_{21}^{2}$ and $m_{3}^{2}=m_{1}^{2}+\Delta m_{31}^{2}$. By projecting $f$ and $g$ onto their absolute values, we obtain two relations between real quantities connecting the mass $m_{1}$ and the PMNS phase $\delta$. Extracting imaginary parts from equations (3.13) and (3.14) gives nontrivial relations between the observable $\delta^{\prime}$ and the PMNS phases, and allows to determine $\alpha$ and $\beta$ in terms of $m_{1}, \delta$, and the known mixing angles and mass squared differences.

In eqs. (3.13) and (3.14) the input parameters are listed in square brackets. Their approximate averages, which for our purpose represent an adequate level of approximation, are reported in table 1. Neutrinos mass squared differences are taken from the global fit in ref. [145] and renormalized to the scale $\Lambda=10^{9} \mathrm{GeV}\left(\sim M_{R}\right)$, with a multiplicative factor $r^{2}(r=1.25$, according to the prescription in ref. [144]). The up-quark masses, renormalized to the scale $\Lambda$, are taken from table IV in ref. [143]. The CKM mixing angles $\theta_{i j}^{\prime}$ and CKM phase $\delta^{\prime}$ are derived from the values of the Wolfenstein parameters given by the PDG [146]. The PMNS mixing angles are taken from the global fit in table 1 of ref. [145], under the assumption of normal hierarchy of the neutrino masses. Renormalization effects for the CKM and PMNS parameters have been neglected. It is worth noting that the $\left|V_{u b}\right|$ puzzle keeps affecting the uncertainty of the small $\theta_{13}^{\prime}$ value. ${ }^{16}$

Given that the signs of $\theta_{12}, \theta_{23}$ and $\theta_{13}$ are not determined in oscillation experiments, depending on the possible choices $\pm \theta_{i j}$ the two eqs. (3.13)-(3.14) represent in principle $2^{3}=8$ conditions. We focus on the case $\left(\theta_{12}, \theta_{23}\right)=\left(-\left|\theta_{12}\right|,-\left|\theta_{23}\right|\right)$, which, according to ref. [36], where an analogous procedure is used in the contest of non-SUSY SO(10) GUT, is a phenomenologically acceptable case.

The plots of $m_{1}$ as a function of $\delta$ are reported in figure 2. The solid and the broken lines correspond to the curves $m_{1}(\delta)$, derived, as explained before, from the two conditions among real parameters obtained by (3.13) and (3.14), respectively. The solutions $\left(m_{1}, \delta\right)$

\footnotetext{
${ }^{16}$ For reviews on the $V_{u b}$ uncertainties see e.g. [150-155].
} 


\begin{tabular}{|c|c|c|c|}
\hline \multicolumn{2}{|c|}{ Quark sector } & \multicolumn{2}{c|}{ Neutrino sector } \\
\hline$m_{u}(\Lambda)$ & $0.00067 \mathrm{GeV}$ & $\Delta m_{21}^{2}(\Lambda)$ & $11.71 \times 10^{-5} \mathrm{eV}^{2}$ \\
$m_{c}(\Lambda)$ & $0.327 \mathrm{GeV}$ & $\Delta m_{31}^{2}(\Lambda)$ & $3.84 \times 10^{-3} \mathrm{eV}^{2}$ \\
$m_{t}(\Lambda)$ & $99.1 \quad \mathrm{GeV}$ & & \\
\hline$\theta_{12}^{\prime}$ & $13.03^{\circ}$ & $\theta_{12}$ & $33.5^{\circ}$ \\
$\theta_{23}^{\prime}$ & $2.37^{\circ}$ & $\theta_{23}$ & $42.3^{\circ}$ \\
$\theta_{13}^{\prime}$ & $0.24^{\circ}$ & $\theta_{13}$ & $8.5^{\circ}$ \\
$\delta^{\prime}$ & $1.19 \mathrm{rad}$ & & \\
\hline
\end{tabular}

Table 1. Input parameters. We use the up-quark masses renormalized to the scale $\Lambda=10^{9} \mathrm{GeV}$ given in table IV in ref. [143]. neutrino's mass squared differences are taken from the global fit in ref. [145] and renormalized to the scale $\Lambda$ with a multiplicative factor $r^{2}$ with $r=1.25$ according to the prescription in ref. [144]. The CKM mixing angles $\theta_{i j}^{\prime}$ and CKM phase $\delta^{\prime}$ are derived from the values of the Wolfenstein parameters given by the PDG [146]. The PMNS mixing angles are taken from the global fit in ref. [145]. Renormalization effects for the CKM and PMNS parameters have been neglected.

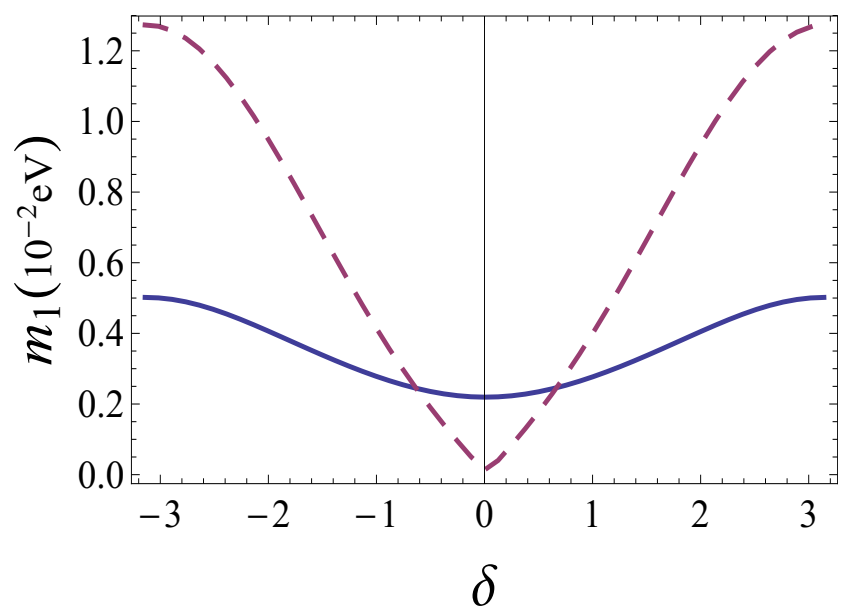

Figure 2. Plots of $m_{1}$ in $m e V$ as a function of $\delta$, when $\left(\theta_{12}, \theta_{23}\right)=\left(-33.5^{\circ},-42.3^{\circ}\right)$. The points of intersections represent possible solutions for $\left(m_{1}, \delta\right)$.

correspond to the intersections between the two lines. Exploiting the constraints on the imaginary parameters given by the same eqs. (3.13)-(3.14) results in predictions for $\alpha$ and $\beta$ as well. Summarizing, the yet-unknown neutrino parameters $m_{1}, \delta, \alpha$ and $\beta$ are given, in our approach, by the following two possibilities

$$
m_{1} \simeq 2.5 \times 10^{-3} \mathrm{eV} \quad \delta \simeq \pm 0.6 \quad \alpha \simeq \mp 1.4 \quad \beta \simeq \mp 0.9
$$

which correspond to the upper or lower sign of the three phases. Current experimental data have recently started to put constraints on the Dirac CP-violating phase and we can compare with a recent result of global $3 \nu$ oscillation analysis which give a $1 \sigma$ range $\delta / \pi \in$ $[1.12,1.77]$ for normal hierarchy [168]. However, at $3 \sigma$, all values $[0,2]$ are still allowed. 


\section{Leptogenesis}

Most of the interest in the values of the masses of $\mathrm{RH}$ neutrinos lies in their double role in the see-saw mechanism and in leptogenesis. Without loss of generality, it is convenient to work in the basis where the RH neutrino mass matrix $M_{R}$ is diagonal. Since $M_{R}$ is symmetric, it can be brought to diagonal form $M_{R}^{\text {diag }}=\operatorname{diag}\left(M_{1}, M_{2}, M_{3}\right)$ with real and positive entries by means of a unitary matrix $W$ :

$$
M_{R}^{\text {diag }}=W^{\dagger} M_{R} W^{*}
$$

We indicate the Dirac mass matrix in this basis as

$$
\hat{m}_{D}=m_{D} W^{*} .
$$

In this section we discuss the same case study of section 3.2 , by setting $\left(\theta_{12}, \theta_{23}\right)=$ $\left(-\left|\theta_{12}\right|,-\left|\theta_{23}\right|\right)$. By arranging the ordering of $\mathrm{RH}$ neutrino masses according to $M_{1}<$ $M_{2}<M_{3}$, our predictions for the RH masses are

$$
M_{1} \simeq 3.5 \times 10^{9} \mathrm{GeV} \quad M_{2} \simeq M_{3} \simeq 8.7 \times 10^{9} \mathrm{GeV}
$$

The numerical differences between the absolute values of each pair of solutions for $\delta$ are negligible. There is no large hierarchy between the masses, and the RH spectrum is compact, with values in the correct range for leptogenesis. Let us observe that the degeneracy of the eigenstates $M_{2} \simeq M_{3}$ is lifted when the condition (3.6) is only approximately satisfied.

The $\mathrm{CP}$ asymmetry in the decay of the $\mathrm{RH}$ neutrino $N_{i}(i=1,2,3)$ to a lepton $\ell_{\alpha}$ $(\alpha=e, \mu, \tau)$ is given by $[156-158]$

$$
\begin{aligned}
\epsilon_{i \alpha}= & \frac{1}{8 \pi v^{2}} \sum_{k \neq i} \frac{\operatorname{Im}\left[\left(\hat{m}_{D}^{\dagger}\right)_{i \alpha}\left(\hat{m}_{D}\right)_{\alpha k}\left(\hat{m}_{D}^{\dagger} \hat{m}_{D}\right)_{i k}\right]}{\left(\hat{m}_{D}^{\dagger} \hat{m}_{D}\right)_{i i}} f_{L V}\left(\frac{M_{k}^{2}}{M_{i}^{2}}\right) \\
& +\frac{1}{8 \pi v^{2}} \sum_{k \neq i} \frac{\operatorname{Im}\left[\left(\hat{m}_{D}^{\dagger}\right)_{i \alpha}\left(\hat{m}_{D}\right)_{\alpha k}\left(\hat{m}_{D}^{\dagger} \hat{m}_{D}\right)_{k i}\right]}{\left(\hat{m}_{D}^{\dagger} \hat{m}_{D}\right)_{i i}} f_{L C}\left(\frac{M_{k}^{2}}{M_{i}^{2}}\right),
\end{aligned}
$$

where $v=174 \mathrm{GeV}$ is the EW VEV. The loop functions are

$$
\begin{aligned}
& f_{L V}(x)=\sqrt{x}\left[\frac{1-x}{(1-x)^{2}+\left(\frac{\Gamma_{i}}{M_{i}}-x \frac{\Gamma_{k}}{M_{k}}\right)^{2}}+1-(1+x) \log \frac{1+x}{x}\right], \\
& f_{L C}(x)=\frac{1-x}{(1-x)^{2}+\left(\frac{\Gamma_{i}}{M_{i}}-x \frac{\Gamma_{k}}{M_{k}}\right)^{2}},
\end{aligned}
$$

where

$$
\Gamma_{i} \equiv \frac{M_{i}}{8 \pi v^{2}}\left(\hat{m}_{D}^{\dagger} \hat{m}_{D}\right)_{i i}
$$




\begin{tabular}{|lll|}
\hline \multicolumn{3}{|c|}{ Washout projectors } \\
\hline$P_{1 e} \simeq 0.02$ & $P_{1 \mu} \simeq 0.42$ & $P_{1 \tau} \simeq 0.56$ \\
$P_{2 e} \simeq 7.40 \times 10^{-5}$ & $P_{2 \mu} \simeq 1.62 \times 10^{-3}$ & $P_{2 \tau} \simeq 0.99$ \\
$P_{3 e} \simeq 7.42 \times 10^{-5}$ & $P_{3 \mu} \simeq 0.42$ & $P_{3 \tau} \simeq 0.99$ \\
\hline & Washout parameters & \\
\hline$\widetilde{m}_{1} \simeq 7.6 \times 10^{-2} \mathrm{eV}$ & $\tilde{m}_{2} \simeq 565 \mathrm{eV}$ & $\widetilde{m}_{3} \simeq 565 \mathrm{eV}$ \\
\hline
\end{tabular}

Table 2. Leptogenesis washout projectors and parameters.

is the total $N_{i}$ width. The first term in eq. (4.4) comes from lepton-number-violating wave and vertex diagrams, while the second term is from the lepton-number-conserving (but lepton-flavour-violating) wave diagram. The rescaled decay width

$$
\widetilde{m}_{i} \equiv \frac{8 \pi v^{2}}{M_{i}^{2}} \Gamma_{i}=\frac{\left(\hat{m}_{D}^{\dagger} \hat{m}_{D}\right)_{i i}}{M_{i}}
$$

which is also known as the effective washout parameter, parameterizes conveniently the departure from thermal equilibrium of $N_{i}$-related processes (the larger $\widetilde{m}_{i}$, the closer to thermal equilibrium the decays and inverse decays of $N_{i}$ occur, thus suppressing the final lepton asymmetry).

The washout projector, $P_{i \alpha}$, projects the decay rate over the $\alpha$ flavour, that is, it corresponds to the branching ratio for $N_{i}$ decaying to $\ell_{\alpha}$, and can be written as

$$
P_{i \alpha}=\frac{\left(\hat{m}_{D}^{\dagger}\right)_{i \alpha}\left(\hat{m}_{D}\right)_{\alpha i}}{\left(\hat{m}_{D}^{\dagger} \hat{m}_{D}\right)_{i i}} .
$$

Finally, the combination $P_{i \alpha} \widetilde{m}_{i}$ projects the washout parameter over a particular flavour direction, and determines how strongly the lepton asymmetry of flavour $\alpha$ is washed out.

Our results for the washout projectors and parameters are collected in table 2, given the values found in eq. (3.15) (differences for $\delta>0$ or $\delta<0$ are negligible). Our results for the $\mathrm{CP}$ asymmetries are collected in table 3 , for positive and negative values of $\delta$, respectively.

In order to calculate the baryon asymmetry, we need to solve a set of Boltzmann equations (BE) derived as in ref. [36]. We report here such derivation for convenience's sake. By including for simplicity only decays and inverse decays, the BE for the $\mathrm{RH}$ neutrino densities $Y_{N_{i}}$ and for $Y_{\Delta_{\alpha}}$, that is the asymmetry density of the charge $B / 3-L_{\alpha}$ normalized to the entropy density $s$, take the form:

$$
\begin{aligned}
& s H z \frac{d Y_{N_{i}}}{d z}=-\gamma_{N_{i}}\left(\frac{Y_{N_{i}}}{Y_{N}^{\mathrm{eq}}}-1\right), \\
& s H z \frac{d Y_{\Delta_{\alpha}}}{d z}=-\sum_{i}\left[\epsilon_{i \alpha} \gamma_{N_{i}}\left(\frac{Y_{N_{i}}}{Y_{N}^{\mathrm{eq}}}-1\right)-\frac{\gamma_{N_{i \alpha}}}{2}\left(\frac{Y_{\Delta \ell_{\alpha}}}{Y_{\ell}^{\mathrm{eq}}}+\frac{Y_{\Delta H}}{Y_{H}^{\mathrm{eq}}}\right)\right],
\end{aligned}
$$




\begin{tabular}{|lll|}
\hline \multicolumn{3}{|c|}{ CP asymmetries } \\
\hline$\epsilon_{1 e} \simeq(-0.13,-0.03) \times 10^{-5}$ & $\epsilon_{1 \mu} \simeq(-1.02,1.39) \times 10^{-5}$ & $\epsilon_{1 \tau} \simeq(1.16,-1.37) \times 10^{-5}$ \\
$\epsilon_{2 e} \simeq(0.67,-1.01) \times 10^{-9}$ & $\epsilon_{2 \mu} \simeq(1.77,-1.88) \times 10^{-8}$ & $\epsilon_{2 \tau} \simeq(1.23,-1.31) \times 10^{-5}$ \\
$\epsilon_{3 e} \simeq(0.70,-1.02) \times 10^{-9}$ & $\epsilon_{3 \mu} \simeq(1.85,-1.91) \times 10^{-8}$ & $\epsilon_{3 \tau} \simeq(1.23,-1.31) \times 10^{-5}$ \\
\hline
\end{tabular}

Table 3. CP asymmetries, The first and second values in parenthesis refer to positive and negative values of $\delta$, respectively, as given by eq. (3.15).

where $Y_{N}^{\mathrm{eq}}=\frac{45}{4 \pi^{4} g_{*}} z^{2} \mathcal{K}_{2}(z)$ is the equilibrium density for the RH neutrinos with $g_{*}=106.75$ and $\mathcal{K}_{2}$ the second order modified Bessel function of the second kind, $2 Y_{\ell}^{\mathrm{eq}}=Y_{H}^{\mathrm{eq}}=\frac{15}{4 \pi^{2} g_{*}}$ are respectively the equilibrium densities for lepton doublets and for the Higgs, and the integration variable is $z=M / T$ with $T$ the temperature of the thermal bath. Here $Y_{\Delta_{\alpha}} \equiv$ $Y_{\Delta B} / 3-Y_{\Delta L_{\alpha}}$ where $Y_{\Delta L_{\alpha}}$ is the total lepton density asymmetry in the $\alpha$ flavour which also includes the asymmetries in the $\mathrm{RH}$ lepton singlets. Since $\mathrm{RH}$ neutrinos only interact with lepton doublets, the right hand side of the second equation of eqs. (4.9) involves only the LH lepton doublets density asymmetry in a given flavour $\alpha, Y_{\Delta \ell_{\alpha}}=A_{\alpha \beta} Y_{\Delta_{\alpha}}$ with $A_{\alpha \beta}$ the flavour mixing matrix $[160,161]$ given in eq. (4.10). In equation (4.9) it is also used $Y_{\Delta H}=C_{\beta} Y_{\Delta_{\beta}}$ the Higgs density asymmetry with $C_{\beta}$ [162] given in (4.10) and $\gamma_{N_{i \alpha}}=P_{i \alpha} \gamma_{N_{i}}$ (no sum over $i$ ). The $A$ flavour mixing matrix and the $C$ vectors in the relevant temperature regime are given by [163]

$$
\begin{aligned}
& A=\frac{1}{2148}\left(\begin{array}{ccc}
-906 & 120 & 120 \\
75 & -688 & 28 \\
75 & 28 & -688
\end{array}\right), \\
& C=-\frac{1}{358}(37,52,52) .
\end{aligned}
$$

We have solved numerically the BE in eq. (4.9) and found the baryon asymmetry generated through leptogenesis according to the relation [164]

$$
Y_{\Delta B}=\frac{28}{79} \sum_{\alpha} Y_{\Delta_{\alpha}}
$$

Our average result is

$$
Y_{\Delta B} \simeq 2.19 \times 10^{-10}
$$

which correspond to the input parameters in eq. (3.15) with positive $\delta$. By comparing with experimental data, we find it sufficiently close to the experimental value to be phenomenologically acceptable. Indeed, recent combined Planck and WMAP CMB measurements $[166,167]$ yield, at $95 \%$ c.l.

$$
Y_{\Delta B}^{\mathrm{P} / \mathrm{WMAP}}=(8.58 \pm 0.22) \times 10^{-11} .
$$

Let us underline that it is not a trivial result to recover the sign and the order of magnitude of the experimental data, given the high degree of predictability of our model. 
Comparison with data allows us to discard the second possibility granted by (3.15), corresponding to $\delta<0$, which results in a negative value $Y_{\Delta B} \simeq-0.23 \times 10^{-11}$. Let us observe that a small difference of input parameters can have a non negligible impact on the values of leptogenesis asymmetries, in contrast to what happens for the values of masses $m_{1}$ and $M_{i}$.

\section{Phenomenology in neutron-antineutron physics}

The mass matrix $M_{\mathrm{RH}}^{N P}$ has to have eigenvalues smaller than the LR symmetry breaking scale $v_{R}$ :

$$
M_{\mathrm{RH}, 1,2,3}^{E 2}<v_{1,2}<v_{R}
$$

On the other hand, we have assumed that

$$
M_{\mathrm{RH}, 1,2,3}^{E 2} \gg \frac{v_{1} v_{2}}{M_{F 2}}
$$

So, the scale $M_{F 2}$ has to be $M_{F 2} \gg 10^{9} \mathrm{GeV}$. This case is compatible with the natural situation $M_{F 2} \simeq M_{S} \cdot{ }^{17}$

On the other hand, the string scale has necessary to be higher than the RH neutrino mass, i.e. $M_{S}>10^{9} \mathrm{GeV}$. These bounds have important implications for other signatures in phenomenology.

Neutron-antineutron transitions generated by new physics at a scale $300 \div 1000 \mathrm{TeV}$ can be tested in the next generation of experiments. In particular the AB-model predicts this signature, even if the precise scale is unknown. The strength of neutron-antineutron transitions is

$$
G_{n-\bar{n}} \simeq \frac{g_{3}^{2}}{16 \pi} \frac{f_{11}^{2} v_{2}}{M_{\Delta_{u^{c} u^{c}}^{c}}^{2} M_{\Delta_{d^{c} d^{c}}^{c}}^{2} M_{\mathrm{SUSY}} \mathcal{M}_{0}^{\prime}}
$$

where $f_{11}=\tilde{f}_{11} v_{1} / M_{2}$ with $\tilde{f}_{11}$ Yukawa couplings $\tilde{f}_{11} v_{1} Q^{c} Q^{c} \Delta^{c} / M_{F 2}$, including $f_{11} \Delta_{u^{c} u^{c}}^{c} u^{c} u^{c}$ and $f_{11} \Delta_{d^{c} d^{c}}^{c} d^{c} d^{c} ; \Delta_{u^{c} u^{c}}, \Delta_{d^{c} d^{c}}$ are the sextets contained in $\Delta^{c}$. This can be rewritten as the following bound on the sextets

$$
\frac{1}{f_{11}^{2}} M_{\Delta_{u^{c} u^{c}}}^{2} M_{\Delta_{d^{c} d^{c}}}^{2}>\frac{(300 \mathrm{TeV})^{5} v_{2}}{M_{\mathrm{SUSY}} M_{S} e^{-S_{E 2^{\prime}}}}
$$

A conservative assumption on the sextets, in order to avoid FCNCs bounds, is $M_{\Delta_{u^{c} u^{c}}} \simeq$ $M_{\Delta_{d^{c} d^{c}}}>100 \mathrm{TeV}$ (with $f_{11} \simeq 1$ ). Calling $x=v_{2} / M_{\mathrm{SUSY}}$, FCNCs bounds will constrains $M_{S}, e^{-S_{E 2^{\prime}}}, x$ as

$$
x^{-1} M_{S} e^{+S_{E 2}^{\prime}}>100 \mathrm{TeV}
$$

at system with $M_{\mathrm{SUSY}}>10^{9} \mathrm{GeV}, v_{1,2}<v_{R}$ and $M_{\mathrm{SUSY}} \leq M_{S}$. These bounds correspond to several different regions of the parameters space, compatible with neutrino physics. As a consequence, our model provides a viable way to generate a Majorana mass for the neutron testable in the next generation of experiments. ${ }^{18}$ On the the other hand, the

\footnotetext{
${ }^{17}$ As a consequence, our model is not compatible with a TeV-ish LR symmetric model.

${ }^{18}$ Neutron-Antineutron transitions could be also an intriguing test for new interactions, as discuss in [149].
} 
generation of such a $B-L$ violating operator can be dangerous in combination with $B+L$ violating sphalerons: they can wash-out an initial lepton number asymmetry generated by RH neutrinos decays. Of course, they can regenerate the correct amount of baryon asymmetry through a post-sphaleron mechanism, as discussed in $[6,18]$. On the other hand, from a string theory prospective, it is reasonable to consider the case in which the strength of the effective operators coupling six quarks increases as a dynamical field from the early Universe to the present epoch. Moduli stabilization is one of the most challenging problem in string theory, because it necessary involves non-perturbative effects such as fluxes and stringy instantons. In string theory, coupling constants, such $\alpha_{e m}$ and so on, are functions of dynamical moduli $f\left(\phi_{i}\right)$, that in turn have to be somehow stabilized. However, in principle, moduli can undergo a slow cosmological evolution rather than being exactly constant in time. As a result, a slowly growing coupling can be naturally envisaged in string inspired models. A natural ansatz can be a solitonic solution in time connecting to constant asymptotes. The naturalness of such a proposal is also supported by the fact that usually the dependence of coupling constants on moduli is of exponential type. In our case, we can suggest a solitonic solution growing from $G_{n \bar{n}}\left(t \ll t_{e . w}\right) \ll \bar{G}_{n \bar{n}}\left(t_{e . w} \ll \bar{t} \ll t_{\mathrm{BBN}}\right)$ to $\bar{G}_{n \bar{n}}$, where $\bar{G}_{n \bar{n}}$ is bounded by direct laboratory limits. Under this general assumption, we also avoid cosmological limits from BBN (Big Bang Nucleosynthesis). Let us remark that the moduli dependence of $G_{n \bar{n}}$ could enter from the non-perturbative mixing of 10plets $\Delta$, i.e. in instantonic geometric moduli. Of course, such a proposal deserves future investigations in global stringy models, beyond the purposes of this paper.

\section{Conclusions and remarks}

In this paper, we have considered an alternative see-saw mechanism produced by exotic instantons rather than by spontaneous symmetry breaking. We have named this mechanism "exotic see-saw" mechanism, since exotic instantons generate the main contribution to the mass matrix of RH neutrinos. We have embedded such a mechanism in an (un)oriented string model with intersecting D-branes and E-branes, giving rise to a Pati-Salam like model in the low energy limit, plus extra non-perturbative couplings. The specific unoriented quiver theory that we have considered was largely inspired by the one suggested in [47]. The present model has a predictive power in low energy observables, not common to other see-saw models.

Our model makes precise predictions for low energy physics, from the acquisition of 11 inputs from neutrino physics. Seven degrees of freedom parameterize the geometry of the mixed disk amplitudes, i.e. of E2-instanton intersecting D6-branes' stacks. We have reconstructed the seven geometric parameters associated to the exotic instanton and we have predictions to compare with the next generation of experiments. This will allow to indirectly test if the E2-instanton considered really dominates the mass terms in the neutrino sector. We have considered a class of mixed disk amplitudes producing a $\mathrm{RH}$ neutrino mass matrix with quasi degenerate spectrum of eigenvalues. The compactness of the RH neutrino spectrum is geometrically understood in terms of mixed disk amplitudes and it is a favorable feature for predictability. As shown, this mechanism can also realize 
a successful baryogenesis through RH neutrinos decays. In our model, a $\theta_{13} \neq 0$ is compatible with leptogenesis and other neutrino physics bounds. Our model is also suggesting other possible signatures in neutron-antineutron transitions [47]. On the other hand, our model is assuming a supersymmetry breaking scale $M_{\text {SUSY }} \gg 1 \mathrm{TeV}$ as well as a Left-Right symmetry scale $M_{L R} \gg 1 \mathrm{TeV}$. A possible discover of Supersymmetry of Left-Right symmetry at LHC or future high energy colliders would rule out our model. In conclusion, our model provides a unifying picture of particles and interactions that will be indirectly tested from different low energy channels in neutrino physics, flavor changing neutral currents, neutron-antineutron transitions and LHC.

\section{Acknowledgments}

It is a pleasure to acknowledge interesting conversations with the participants in the XIV Marcel Grossmann Meeting in Roma (12-18 July 2015) during which this project was carried on. G. R. thanks C. S. Fong for very useful and interesting discussions. The work of A. A. was supported in part by the MIUR research grant "Theoretical Astroparticle Physics" PRIN 2012CPPYP7. The work of M. B. was partly supported by the INFN network "ST\&FI" and by the Uncovering Excellence Grant "STaI" of the University of Rome "Tor Vergata". The work of G. R. was supported in part by MIUR under project 2010YJ2NYW and INFN under specific initiative QNP.

Open Access. This article is distributed under the terms of the Creative Commons Attribution License (CC-BY 4.0), which permits any use, distribution and reproduction in any medium, provided the original author(s) and source are credited.

\section{References}

[1] E. Majorana, Theory of the Symmetry of Electrons and Positrons, Nuovo Cim. 14 (1937) 171 [INSPIRE].

[2] P. Minkowski, $\mu \rightarrow$ er at a Rate of One Out of $10^{9}$ Muon Decays?, Phys. Lett. B 67 (1977) 421 [INSPIRE].

[3] P. Ramond, The Family Group in Grand Unified Theories, in Sanibel Conference, CALT-68-700 (1979), [hep-ph/9809459] [INSPIRE].

[4] M. Gell-Mann, P. Ramond and R. Slansky, Complex Spinors and Unified Theories, in Supergravity (1979), Print 80-0576, [Conf. Proc. C 790927 (1979) 315] [arXiv:1306.4669] [INSPIRE].

[5] T. Yanagida, Horizontal Symmetry and Masses of Neutrinos, Prog. Theor. Phys. 64 (1980) 1103 [INSPIRE].

[6] R.N. Mohapatra and G. Senjanović, Neutrino Mass and Spontaneous Parity Violation, Phys. Rev. Lett. 44 (1980) 912 [inSPIRE].

[7] M. Fukugita and T. Yanagida, Baryogenesis Without Grand Unification, Phys. Lett. B 174 (1986) 45 [INSPIRE]. 
[8] A.D. Sakharov, Violation of CP Invariance, c Asymmetry and Baryon Asymmetry of the Universe, Pisma Zh. Eksp. Teor. Fiz. 5 (1967) 32 [JETP Lett. 5 (1967) 24] [Sov. Phys. Usp. 34 (1991) 392] [Usp. Fiz. Nauk 161 (1991) 61] [InSPIRE].

[9] G. Chen, M.J. Pérez and P. Ramond, Neutrino masses, the $\mu$-term and $\mathcal{P} \mathcal{S} \mathcal{L}_{2}(7)$, Phys. Rev. D 92 (2015) 076006 [arXiv: 1412.6107] [InSPIRE].

[10] J. Kile, M.J. Pérez, P. Ramond and J. Zhang, $\theta_{13}$ and the flavor ring, Phys. Rev. D 90 (2014) 013004 [arXiv: 1403.6136] [INSPIRE].

[11] Y. Mambrini, N. Nagata, K.A. Olive, J. Quevillon and J. Zheng, Dark matter and gauge coupling unification in nonsupersymmetric $\mathrm{SO}(10)$ grand unified models, Phys. Rev. D 91 (2015) 095010 [arXiv: 1502.06929] [INSPIRE].

[12] J. Hisano, Y. Muramatsu, Y. Omura and M. Yamanaka, Flavor violating $Z^{\prime}$ from $\mathrm{SO}(10)$ SUSY GUT in High-Scale SUSY, Phys. Lett. B 744 (2015) 395 [arXiv:1503.06156] [INSPIRE].

[13] F. Wang, W. Wang and J.M. Yang, A split SUSY model from SUSY GUT, JHEP 03 (2015) 050 [arXiv: 1501.02906] [INSPIRE].

[14] G. 't Hooft, Computation of the Quantum Effects Due to a Four-Dimensional Pseudoparticle, Phys. Rev. D 14 (1976) 3432 [Erratum ibid. D 18 (1978) 2199] [INSPIRE].

[15] V.A. Kuzmin, V.A. Rubakov and M.E. Shaposhnikov, On the Anomalous Electroweak Baryon Number Nonconservation in the Early Universe, Phys. Lett. B 155 (1985) 36 [INSPIRE].

[16] J.C. Pati and A. Salam, Lepton Number as the Fourth Color, Phys. Rev. D 10 (1974) 275 [Erratum ibid. D 11 (1975) 703] [INSPIRE].

[17] S. Dimopoulos and F. Wilczek, Supersymmetric Unified Models, in Erice 1981, Proceedings, The Unity Of The Fundamental Interactions, A. Zichichi ed., Conf. Proc. C 810731 (1981) 237.

[18] K.S. Babu and R.N. Mohapatra, Mass matrix textures from superstring inspired $\mathrm{SO}(10)$ models, Phys. Rev. Lett. 74 (1995) 2418 [hep-ph/9410326] [INSPIRE].

[19] Z. Berezhiani and G. Dvali, Possible solution of the hierarchy problem in supersymmetrical grand unification theories, Bull. Lebedev Phys. Inst. 5 (1989) 55 [Kratk. Soobshch. Fiz. 5 (1989) 42] [INSPIRE].

[20] R. Barbieri, G.R. Dvali and A. Strumia, Grand unified supersymmetric Higgs bosons as pseudoGoldstone particles, Nucl. Phys. B 391 (1993) 487 [InSPIRE].

[21] L. Randall and C. Csáki, The doublet-triplet splitting problem and Higgses as pseudoGoldstone bosons, in Palaiseau 1995, SUSY 95, hep-ph/9508208 [INSPIRE].

[22] P. Candelas, G.T. Horowitz, A. Strominger and E. Witten, Vacuum Configurations for Superstrings, Nucl. Phys. B 258 (1985) 46 [InSPIRE].

[23] L.J. Dixon, J.A. Harvey, C. Vafa and E. Witten, Strings on Orbifolds, Nucl. Phys. B 261 (1985) 678 [INSPIRE].

[24] L.J. Dixon, J.A. Harvey, C. Vafa and E. Witten, Strings on Orbifolds. 2., Nucl. Phys. B 274 (1986) 285 [INSPIRE].

[25] L.E. Ibáñez, H.P. Nilles and F. Quevedo, Orbifolds and Wilson Lines, Phys. Lett. B 187 (1987) 25 [INSPIRE]. 
[26] L.E. Ibáñez, J.E. Kim, H.P. Nilles and F. Quevedo, Orbifold Compactifications with Three Families of $\mathrm{SU}(3) \times \mathrm{SU}(2) \times \mathrm{U}(1)^{n}$, Phys. Lett. B 191 (1987) 282 [inSPIRE].

[27] S. Davidson and A. Ibarra, A lower bound on the right-handed neutrino mass from leptogenesis, Phys. Lett. B 535 (2002) 25 [hep-ph/0202239] [INSPIRE].

[28] G. Engelhard, Y. Grossman, E. Nardi and Y. Nir, The importance of N2 leptogenesis, Phys. Rev. Lett. 99 (2007) 081802 [hep-ph/0612187] [INSPIRE].

[29] P. Di Bari, Seesaw geometry and leptogenesis, Nucl. Phys. B 727 (2005) 318 [hep-ph/0502082] [INSPIRE].

[30] O. Vives, Flavor dependence of CP asymmetries and thermal leptogenesis with strong right-handed neutrino mass hierarchy, Phys. Rev. D 73 (2006) 073006 [hep-ph/0512160] [INSPIRE].

[31] A. Abada, P. Hosteins, F.-X. Josse-Michaux and S. Lavignac, Successful Leptogenesis in SO(10) Unification with a Left-Right Symmetric Seesaw Mechanism, Nucl. Phys. B 809 (2009) 183 [arXiv: 0808.2058] [INSPIRE].

[32] P. Di Bari and A. Riotto, Successful type-I Leptogenesis with $\mathrm{SO}(10)$-inspired mass relations, Phys. Lett. B 671 (2009) 462 [arXiv:0809.2285] [INSPIRE].

[33] P. Di Bari and A. Riotto, Testing $\mathrm{SO}(10)$-inspired leptogenesis with low energy neutrino experiments, JCAP 04 (2011) 037 [arXiv: 1012.2343] [INSPIRE].

[34] S. Blanchet, D. Marfatia and A. Mustafayev, Examining leptogenesis with lepton flavor violation and the dark matter abundance, JHEP 11 (2010) 038 [arXiv:1006.2857] [INSPIRE].

[35] F. Buccella, D. Falcone and L. Oliver, Baryogenesis via leptogenesis from quark-lepton symmetry and a compact heavy $N_{R}$ spectrum, Phys. Rev. D 83 (2011) 093013 [arXiv:1006.5698] [INSPIRE].

[36] F. Buccella, D. Falcone, C.S. Fong, E. Nardi and G. Ricciardi, Squeezing out predictions with leptogenesis from $\mathrm{SO}(10)$, Phys. Rev. D 86 (2012) 035012 [arXiv:1203.0829] [inSPIRE].

[37] A. Pilaftsis and T.E.J. Underwood, Resonant leptogenesis, Nucl. Phys. B 692 (2004) 303 [hep-ph/0309342] [INSPIRE].

[38] A. Pilaftsis and T.E.J. Underwood, Electroweak-scale resonant leptogenesis, Phys. Rev. D 72 (2005) 113001 [hep-ph/0506107] [INSPIRE].

[39] A. Pilaftsis, Resonant tau-leptogenesis with observable lepton number violation, Phys. Rev. Lett. 95 (2005) 081602 [hep-ph/0408103] [INSPIRE].

[40] L. Marzola, Strong thermal Leptogenesis: an exploded view of the low energy neutrino parameters in the $\mathrm{SO}(10)$-inspired model, arXiv:1410.5565 [INSPIRE].

[41] P. Di Bari, L. Marzola and M. Re Fiorentin, Decrypting SO(10)-inspired leptogenesis, Nucl. Phys. B 893 (2015) 122 [arXiv:1411.5478] [INSPIRE].

[42] C.S. Fong, D. Meloni, A. Meroni and E. Nardi, Leptogenesis in $\mathrm{SO}(10)$, JHEP 01 (2015) 111 [arXiv: 1412.4776] [INSPIRE].

[43] R.N. Mohapatra and R.E. Marshak, Local B-L Symmetry of Electroweak Interactions, Majorana Neutrinos and Neutron Oscillations, Phys. Rev. Lett. 44 (1980) 1316 [Erratum ibid. 44 (1980) 1643] [INSPIRE]. 
[44] F. Quevedo, Local String Models and Moduli Stabilisation, Mod. Phys. Lett. A 30 (2015) 1530004 [arXiv: 1404.5151] [INSPIRE].

[45] S. Krippendorf, M.J. Dolan, A. Maharana and F. Quevedo, D-branes at Toric Singularities: Model Building, Yukawa Couplings and Flavour Physics, JHEP 06 (2010) 092 [arXiv: 1002.1790] [INSPIRE].

[46] J.P. Conlon, A. Maharana and F. Quevedo, Towards Realistic String Vacua, JHEP 05 (2009) 109 [arXiv: 0810.5660] [inSPIRE].

[47] A. Addazi and M. Bianchi, Neutron Majorana mass from Exotic Instantons in a Pati-Salam model, JHEP 06 (2015) 012 [arXiv:1502.08041] [INSPIRE].

[48] P. Anastasopoulos, G.K. Leontaris and N.D. Vlachos, Phenomenological Analysis of D-brane Pati-Salam Vacua, JHEP 05 (2010) 011 [arXiv: 1002.2937] [INSPIRE].

[49] M. Bianchi and M. Samsonyan, Notes on unoriented D-brane instantons, Int. J. Mod. Phys. A 24 (2009) 5737 [arXiv:0909.2173] [InSPIRE].

[50] M. Bianchi and G. Inverso, Unoriented D-brane instantons, Fortsch. Phys. 60 (2012) 822 [arXiv:1202.6508] [INSPIRE].

[51] S. Franco, A. Retolaza and A. Uranga, D-brane Instantons as Gauge Instantons in Orientifolds of Chiral Quiver Theories, JHEP 11 (2015) 165 [arXiv:1507.05330] [INSPIRE].

[52] Particle Data Group collaboration, J. Beringer et al., Review of Particle Physics (RPP), Phys. Rev. D 86 (2012) 010001 [inSPIRE].

[53] M. Baldo-Ceolin et al., A new experimental limit on neutron-antineutron oscillations, $Z$. Phys. C 63 (1994) 409 [INSPIRE].

[54] K.S. Babu et al., Working Group Report: Baryon Number Violation, arXiv:1311.5285 [INSPIRE].

[55] K. Babu et al., Neutron-Antineutron Oscillations: A Snowmass 2013 White Paper, arXiv: 1310.8593 [INSPIRE].

[56] U. Al-Binni et al., Project X: Physics Opportunities, arXiv:1306.5009 [INSPIRE].

[57] K.S. Babu, R.N. Mohapatra and S. Nasri, Unified TeV Scale Picture of Baryogenesis and Dark Matter, Phys. Rev. Lett. 98 (2007) 161301 [hep-ph/0612357] [INSPIRE].

[58] K.S. Babu, R.N. Mohapatra and S. Nasri, Post-Sphaleron Baryogenesis, Phys. Rev. Lett. 97 (2006) 131301 [hep-ph/0606144] [INSPIRE].

[59] K.S. Babu, P.S. Bhupal Dev and R.N. Mohapatra, Neutrino mass hierarchy, neutron-antineutron oscillation from baryogenesis, Phys. Rev. D 79 (2009) 015017 [arXiv: 0811.3411] [INSPIRE].

[60] K.S. Babu, P.S. Bhupal Dev, E.C. F.S. Fortes and R.N. Mohapatra, Post-Sphaleron Baryogenesis and an Upper Limit on the Neutron-Antineutron Oscillation Time, Phys. Rev. D 87 (2013) 115019 [arXiv:1303.6918] [INSPIRE].

[61] E.C. F.S. Fortes, K.S. Babu and R.N. Mohapatra, Flavor Physics Constraints on TeV Scale Color Sextet Scalars, arXiv:1311.4101 [INSPIRE].

[62] R.N. Mohapatra, N. Okada and H.-B. Yu, Diquark Higgs at LHC, Phys. Rev. D 77 (2008) 011701 [arXiv: 0709.1486] [INSPIRE]. 
[63] Y.C. Zhan, Z.L. Liu, S.A. Li, C.S. Li and H.T. Li, Threshold resummation for the production of a color sextet (antitriplet) scalar at the LHC, Eur. Phys. J. C 74 (2014) 2716 [arXiv:1305.5152] [INSPIRE].

[64] A. Addazi and M. Bianchi, Neutron Majorana mass from exotic instantons, JHEP 12 (2014) 089 [arXiv: 1407.2897] [INSPIRE].

[65] A. Addazi, 'Exotic vector-like pair' of color-triplet scalars, JHEP 04 (2015) 153 [arXiv: 1501.04660] [INSPIRE].

[66] A. Addazi and M. Bianchi, Un-oriented Quiver Theories for Majorana Neutrons, JHEP 07 (2015) 144 [arXiv: 1502.01531] [InSPIRE].

[67] A. Addazi, More about Neutron Majorana mass from Exotic Instantons: an alternative mechanism in Low-Scale String theory, arXiv:1504.06799 [INSPIRE].

[68] A. Addazi, Dynamical R-parity violations from exotic instantons, arXiv:1505.00625 [INSPIRE].

[69] A. Addazi, Neutron-antineutron transition as a test-bed for dynamical CPT violations, arXiv: 1505.02080 [INSPIRE].

[70] A. Addazi, Direct generation of a Majorana mass for the Neutron from Exotic Instantons, arXiv: 1506.06351 [INSPIRE].

[71] T. Schwetz, M. Tortola and J.W.F. Valle, Where we are on $\theta_{13}$ : addendum to 'Global neutrino data and recent reactor fluxes: status of three-flavour oscillation parameters', New J. Phys. 13 (2011) 109401 [arXiv:1108.1376] [INSPIRE].

[72] G.L. Fogli, E. Lisi, A. Marrone, A. Palazzo and A.M. Rotunno, Evidence of $\theta_{13}>0$ from global neutrino data analysis, Phys. Rev. D 84 (2011) 053007 [arXiv:1106.6028] [InSPIRE].

[73] M.C. Gonzalez-Garcia, M. Maltoni and J. Salvado, Updated global fit to three neutrino mixing: status of the hints of $\theta_{13}>0$, JHEP 04 (2010) 056 [arXiv: 1001.4524] [INSPIRE].

[74] A. Sagnotti, Open Strings and their Symmetry Groups, in Cargese 1987, Proceedings, Nonperturbative Quantum Field Theory, pg. 521, ROM2F-87-025 [hep-th/0208020] [INSPIRE].

[75] M. Bianchi and A. Sagnotti, Open Strings and the Relative Modular Group, Phys. Lett. B 231 (1989) 389 [INSPIRE].

[76] M. Bianchi and A. Sagnotti, The Geometry Of Open String Partition Functions, ROM2F-88-040, C88-06-06.6 [INSPIRE].

[77] M. Bianchi and A. Sagnotti, The Partition Function of the SO(8192) Bosonic String, Phys. Lett. B 211 (1988) 407 [INSPIRE].

[78] A. Sagnotti, A note on the Green-Schwarz mechanism in open string theories, Phys. Lett. B 294 (1992) 196 [hep-th/9210127] [INSPIRE].

[79] C. Angelantonj and A. Sagnotti, Open strings, Phys. Rept. 371 (2002) 1 [hep-th/0204089] [INSPIRE].

[80] G. Pradisi and A. Sagnotti, Open String Orbifolds, Phys. Lett. B 216 (1989) 59 [InSPIRE].

[81] M. Bianchi and A. Sagnotti, On the systematics of open string theories, Phys. Lett. B 247 (1990) 517 [INSPIRE]. 
[82] M. Bianchi and A. Sagnotti, Twist symmetry and open string Wilson lines, Nucl. Phys. B 361 (1991) 519 [INSPIRE].

[83] M. Bianchi, G. Pradisi and A. Sagnotti, Toroidal compactification and symmetry breaking in open string theories, Nucl. Phys. B 376 (1992) 365 [INSPIRE].

[84] A.M. Uranga, D-brane probes, RR tadpole cancellation and k-theory charge, Nucl. Phys. B 598 (2001) 225 [hep-th/0011048] [INSPIRE].

[85] G. Aldazabal, S. Franco, L.E. Ibáñez, R. Rabadán and A.M. Uranga, $D=4$ chiral string compactifications from intersecting branes, J. Math. Phys. 42 (2001) 3103 [hep-th/0011073] [INSPIRE].

[86] M. Bianchi and J.F. Morales, Anomalies \& tadpoles, JHEP 03 (2000) 030 [hep-th/0002149] [INSPIRE].

[87] C. Angelantonj, M. Bianchi, G. Pradisi, A. Sagnotti and Ya. S. Stanev, Chiral asymmetry in four-dimensional open string vacua, Phys. Lett. B 385 (1996) 96 [hep-th/9606169] [INSPIRE].

[88] C. Angelantonj, M. Bianchi, G. Pradisi, A. Sagnotti and Y.S. Stanev, Comments on Gepner models and type-I vacua in string theory, Phys. Lett. B 387 (1996) 743 [hep-th/9607229] [INSPIRE].

[89] R. Blumenhagen, M. Cvetič and T. Weigand, Spacetime instanton corrections in $4 D$ string vacua: The seesaw mechanism for D-brane models, Nucl. Phys. B 771 (2007) 113 [hep-th/0609191] [INSPIRE].

[90] L.E. Ibáñez and A.M. Uranga, Neutrino Majorana Masses from String Theory Instanton Effects, JHEP 03 (2007) 052 [hep-th/0609213] [INSPIRE].

[91] L.E. Ibáñez, A.N. Schellekens and A.M. Uranga, Instanton Induced Neutrino Majorana Masses in CFT Orientifolds with MSSM-like spectra, JHEP 06 (2007) 011 [arXiv: 0704.1079] [INSPIRE].

[92] S. Antusch, L.E. Ibáñez and T. Macrì, Neutrino masses and mixings from string theory instantons, JHEP 09 (2007) 087 [arXiv:0706.2132] [INSPIRE].

[93] R. Blumenhagen, M. Cvetič, D. Lüst, R. Richter and T. Weigand, Non-perturbative Yukawa Couplings from String Instantons, Phys. Rev. Lett. 100 (2008) 061602 [arXiv:0707.1871] [INSPIRE].

[94] M. Bianchi, S. Cremonesi, A. Hanany, J.F. Morales, D.R. Pacifici and R.-K. Seong, Mass-deformed Brane Tilings, JHEP 10 (2014) 27 [arXiv:1408.1957] [INSPIRE].

[95] P. Anastasopoulos, M. Bianchi, E. Dudas and E. Kiritsis, Anomalies, anomalous U(1)'s and generalized Chern-Simons terms, JHEP 11 (2006) 057 [hep-th/0605225] [INSPIRE].

[96] J. De Rydt, J. Rosseel, T.T. Schmidt, A. Van Proeyen and M. Zagermann, Symplectic structure of $N=1$ supergravity with anomalies and Chern-Simons terms, Class. Quant. Grav. 24 (2007) 5201 [arXiv:0705.4216] [INSPIRE].

[97] D. Feldman, Z. Liu and P. Nath, The Stueckelberg Z-prime Extension with Kinetic Mixing and Milli-Charged Dark Matter From the Hidden Sector, Phys. Rev. D 75 (2007) 115001 [hep-ph/0702123] [INSPIRE].

[98] D. Feldman, Z. Liu and P. Nath, The Stueckelberg extension and milli weak and milli charged dark matter, AIP Conf. Proc. 939 (2007) 50 [arXiv: 0705.2924] [InSPIRE]. 
[99] B. Körs and P. Nath, A Stueckelberg extension of the standard model, Phys. Lett. B 586 (2004) 366 [hep-ph/0402047] [INSPIRE].

[100] B. Körs and P. Nath, A Supersymmetric Stueckelberg U(1) extension of the MSSM, JHEP 12 (2004) 005 [hep-ph/0406167] [INSPIRE].

[101] B. Körs and P. Nath, How Stueckelberg extends the standard model and the MSSM, hep-ph/0411406 [INSPIRE].

[102] B. Körs and P. Nath, Aspects of the Stueckelberg extension, JHEP 07 (2005) 069 [hep-ph/0503208] [INSPIRE].

[103] P. Anastasopoulos, F. Fucito, A. Lionetto, G. Pradisi, A. Racioppi and Y.S. Stanev, Minimal Anomalous U(1)-prime Extension of the MSSM, Phys. Rev. D 78 (2008) 085014 [arXiv:0804.1156] [INSPIRE].

[104] C. Corianò, N. Irges and E. Kiritsis, On the effective theory of low scale orientifold string vacua, Nucl. Phys. B 746 (2006) 77 [hep-ph/0510332] [INSPIRE].

[105] M. Bianchi and E. Kiritsis, Non-perturbative and Flux superpotentials for Type I strings on the Z(3) orbifold, Nucl. Phys. B 782 (2007) 26 [hep-th/0702015] [InSPIRE].

[106] B. de Wit, P.G. Lauwers and A. Van Proeyen, Lagrangians of $N=2$ Supergravity-Matter Systems, Nucl. Phys. B 255 (1985) 569 [INSPIRE].

[107] N. Arkani-Hamed, H. Georgi and M.D. Schwartz, Effective field theory for massive gravitons and gravity in theory space, Annals Phys. 305 (2003) 96 [hep-th/0210184] [INSPIRE].

[108] V.A. Rubakov, Lorentz-violating graviton masses: Getting around ghosts, low strong coupling scale and VDVZ discontinuity, hep-th/0407104 [INSPIRE].

[109] S.L. Dubovsky, Phases of massive gravity, JHEP 10 (2004) 076 [hep-th/0409124] [INSPIRE].

[110] A. Addazi and S. Capozziello, External stability for Spherically Symmetric Solutions in Lorentz Breaking Massive Gravity, Int. J. Theor. Phys. 54 (2015) 1818 [arXiv:1407.4840] [INSPIRE].

[111] A. Addazi, Quantum chaos inside Black Holes, arXiv:1508.04054 [INSPIRE].

[112] M. Bianchi and A.V. Santini, String predictions for near future colliders from one-loop scattering amplitudes around D-brane worlds, JHEP 12 (2006) 010 [hep-th/0607224] [INSPIRE].

[113] A. Addazi and G. Esposito, Nonlocal quantum field theory without acausality and nonunitarity at quantum level: is SUSY the key?, Int. J. Mod. Phys. A 30 (2015) 1550103 [arXiv: 1502.01471] [INSPIRE].

[114] A. Addazi, Unitarization and Causalization of Non-local quantum field theories by Classicalization, arXiv:1505.07357 [INSPIRE].

[115] L.E. Ibáñez, A.N. Schellekens and A.M. Uranga, Discrete Gauge Symmetries in Discrete MSSM-like Orientifolds, Nucl. Phys. B 865 (2012) 509 [arXiv:1205.5364] [InSPIRE].

[116] L.E. Ibáñez and G.G. Ross, Discrete gauge symmetries and the origin of baryon and lepton number conservation in supersymmetric versions of the standard model, Nucl. Phys. B $\mathbf{3 6 8}$ (1992) 3 [INSPIRE].

[117] M. Berasaluce-Gonzalez, L.E. Ibáñez, P. Soler and A.M. Uranga, Discrete gauge symmetries in D-brane models, JHEP 12 (2011) 113 [arXiv:1106.4169] [INSPIRE]. 
[118] P. Anastasopoulos, M. Cvetič, R. Richter and P.K.S. Vaudrevange, String Constraints on Discrete Symmetries in MSSM Type II Quivers, JHEP 03 (2013) 011 [arXiv:1211.1017] [INSPIRE].

[119] G. Honecker and W. Staessens, To Tilt or Not To Tilt: Discrete Gauge Symmetries in Global Intersecting D-brane Models, JHEP 10 (2013) 146 [arXiv:1303.4415] [INSPIRE].

[120] H. Abe, K.-S. Choi, T. Kobayashi and H. Ohki, Non-Abelian Discrete Flavor Symmetries from Magnetized/Intersecting Brane Models, Nucl. Phys. B 820 (2009) 317 [arXiv:0904.2631] [INSPIRE].

[121] M. Berasaluce-Gonzalez, P.G. Camara, F. Marchesano, D. Regalado and A.M. Uranga, Non-Abelian discrete gauge symmetries in 4d string models, JHEP 09 (2012) 059 [arXiv:1206.2383] [INSPIRE].

[122] F. Marchesano, D. Regalado and L. Vazquez-Mercado, Discrete flavor symmetries in D-brane models, JHEP 09 (2013) 028 [arXiv: 1306.1284] [INSPIRE].

[123] Y. Hamada, T. Kobayashi and S. Uemura, Flavor structure in D-brane models: Majorana neutrino masses, JHEP 05 (2014) 116 [arXiv:1402.2052] [INSPIRE].

[124] T. Kobayashi, H.P. Nilles, F. Ploger, S. Raby and M. Ratz, Stringy origin of non-Abelian discrete flavor symmetries, Nucl. Phys. B 768 (2007) 135 [hep-ph/0611020] [INSPIRE].

[125] T.P.T. Dijkstra, L.R. Huiszoon and A.N. Schellekens, Chiral supersymmetric standard model spectra from orientifolds of Gepner models, Phys. Lett. B 609 (2005) 408 [hep-th/0403196] [INSPIRE].

[126] T.P.T. Dijkstra, L.R. Huiszoon and A.N. Schellekens, Supersymmetric standard model spectra from RCFT orientifolds, Nucl. Phys. B 710 (2005) 3 [hep-th/0411129] [INSPIRE].

[127] P. Anastasopoulos, T.P.T. Dijkstra, E. Kiritsis and A.N. Schellekens, Orientifolds, hypercharge embeddings and the Standard Model, Nucl. Phys. B 759 (2006) 83 [hep-th/0605226] [INSPIRE].

[128] R. Blumenhagen, Supersymmetric orientifolds of Gepner models, JHEP 11 (2003) 055 [hep-th/0310244] [INSPIRE].

[129] R. Blumenhagen and T. Weigand, Chiral Gepner model orientifolds, hep-th/0408147 [INSPIRE].

[130] R. Blumenhagen and T. Weigand, A note on partition functions of Gepner model orientifolds, Phys. Lett. B 591 (2004) 161 [hep-th/0403299] [InSPIRE].

[131] R. Blumenhagen and T. Weigand, Chiral supersymmetric Gepner model orientifolds, JHEP 02 (2004) 041 [hep-th/0401148] [INSPIRE].

[132] G. Aldazabal, E.C. Andres, M. Leston and C.A. Núñez, Type IIB orientifolds on Gepner points, JHEP 09 (2003) 067 [hep-th/0307183] [INSPIRE].

[133] S. Govindarajan and J. Majumder, Crosscaps in Gepner models and type IIA orientifolds, JHEP 02 (2004) 026 [hep-th/0306257] [INSPIRE].

[134] G. Aldazabal, E.C. Andres and J.E. Juknevich, Particle models from orientifolds at Gepner orbifold points, JHEP 05 (2004) 054 [hep-th/0403262] [INSPIRE].

[135] I. Brunner, K. Hori, K. Hosomichi and J. Walcher, Orientifolds of Gepner models, JHEP 02 (2007) 001 [hep-th/0401137] [INSPIRE]. 
[136] E. Kiritsis, B. Schellekens and M. Tsulaia, Discriminating MSSM families in (free-field) Gepner Orientifolds, JHEP 10 (2008) 106 [arXiv:0809.0083] [INSPIRE].

[137] E. Kiritsis, M. Lennek and B. Schellekens, SU(5) orientifolds, Yukawa couplings, Stringy Instantons and Proton Decay, Nucl. Phys. B 829 (2010) 298 [arXiv:0909.0271] [INSPIRE].

[138] P. Anastasopoulos, G.K. Leontaris and N.D. Vlachos, Phenomenological Analysis of D-brane Pati-Salam Vacua, JHEP 05 (2010) 011 [arXiv: 1002.2937] [INSPIRE].

[139] P. Anastasopoulos, R. Richter and A.N. Schellekens, Discrete symmetries from hidden sectors, JHEP 06 (2015) 189 [arXiv:1502.02686] [INSPIRE].

[140] T. Takagi, On an Algebraic Problem Related to an Analytic Theorem of Carathéodory and Fejér and on an Allied Theorem of Landau, Japanese J. Math. 1 (1924) 83.

[141] B. Pontecorvo, Mesonium and anti-mesonium, Zh. Eksp. Teor. Fiz. 33 (1957) 549 [Sov. Phys. JETP 6 (1957) 429] [INSPIRE].

[142] B. Pontecorvo, Neutrino Experiments and the Problem of Conservation of Leptonic Charge, Zh. Eksp. Teor. Fiz. 53 (1967) 1717 [Sov. Phys. JETP 26 (1968) 984] [INSPIRE].

[143] Z.-z. Xing, H. Zhang and S. Zhou, Updated Values of Running Quark and Lepton Masses, Phys. Rev. D 77 (2008) 113016 [arXiv:0712.1419] [INSPIRE].

[144] G.F. Giudice, A. Notari, M. Raidal, A. Riotto and A. Strumia, Towards a complete theory of thermal leptogenesis in the SM and MSSM, Nucl. Phys. B 685 (2004) 89 [hep-ph/0310123] [INSPIRE].

[145] M.C. Gonzalez-Garcia, M. Maltoni and T. Schwetz, Updated fit to three neutrino mixing: status of leptonic CP-violation, JHEP 11 (2014) 052 [arXiv:1409.5439] [INSPIRE].

[146] Particle Data Group collaboration, K.A. Olive et al., Review of Particle Physics, Chin. Phys. C 38 (2014) 090001 [inSPIRE].

[147] P. Di Bari, L. Marzola and M. Re Fiorentin, Decrypting SO(10)-inspired leptogenesis, Nucl. Phys. B 893 (2015) 122 [arXiv: 1411.5478] [INSPIRE].

[148] A. Addazi et al., DAMA annual modulation effect and asymmetric mirror matter, Eur. Phys. J. C 75 (2015) 400 [arXiv:1507.04317] [INSPIRE].

[149] A. Addazi, Neutron-Antineutron oscillation as a test of a New Interaction, Nuovo Cim. C $\mathbf{0 3 8}$ (2015) 21 [INSPIRE].

[150] G. Ricciardi et al., Flavour, Electroweak Symmetry Breaking and Dark Matter: state of the art and future prospects, Eur. Phys. J. Plus 130 (2015) 209 [arXiv:1507.05029] [InSPIRE].

[151] G. Ricciardi, Status of $\left|V_{c b}\right|$ and $\left|V_{u b}\right|$ CKM matrix elements, AIP Conf. Proc. 1701 (2016) 050014 [arXiv: 1412.4288] [INSPIRE].

[152] G. Ricciardi, Progress on semi-leptonic B(s) decays, Mod. Phys. Lett. A 29 (2014) 1430019 [arXiv: 1403.7750] [INSPIRE].

[153] G. Ricciardi, Determination of the CKM matrix elements $\left|V_{x b}\right|$, Mod. Phys. Lett. A 28 (2013) 1330016 [arXiv: 1305.2844] [INSPIRE].

[154] G. Ricciardi, Magnitudes of $V_{x b}$ CKM matrix elements, PoS (Beauty 2013) 040 [arXiv:1306.1039] [INSPIRE].

[155] G. Ricciardi, Brief review on semileptonic B decays, Mod. Phys. Lett. A 27 (2012) 1230037 [arXiv: 1209.1407] [INSPIRE]. 
[156] L. Covi, E. Roulet and F. Vissani, CP violating decays in leptogenesis scenarios, Phys. Lett. B 384 (1996) 169 [hep-ph/9605319] [INSPIRE].

[157] W. Buchmüller and M. Plümacher, CP asymmetry in Majorana neutrino decays, Phys. Lett. B 431 (1998) 354 [hep-ph/9710460] [INSPIRE].

[158] A. Anisimov, A. Broncano and M. Plümacher, The CP-asymmetry in resonant leptogenesis, Nucl. Phys. B 737 (2006) 176 [hep-ph/0511248] [INSPIRE].

[159] S. Davidson, E. Nardi and Y. Nir, Leptogenesis, Phys. Rept. 466 (2008) 105 [arXiv: 0802.2962] [INSPIRE].

[160] R. Barbieri, P. Creminelli, A. Strumia and N. Tetradis, Baryogenesis through leptogenesis, Nucl. Phys. B 575 (2000) 61 [hep-ph/9911315] [InSPIRE].

[161] T. Endoh, T. Morozumi and Z.-h. Xiong, Primordial lepton family asymmetries in seesaw model, Prog. Theor. Phys. 111 (2004) 123 [hep-ph/0308276] [InSPIRE].

[162] E. Nardi, Y. Nir, J. Racker and E. Roulet, On Higgs and sphaleron effects during the leptogenesis era, JHEP 01 (2006) 068 [hep-ph/0512052] [INSPIRE].

[163] E. Nardi, Y. Nir, E. Roulet and J. Racker, The importance of flavor in leptogenesis, JHEP 01 (2006) 164 [hep-ph/0601084] [INSPIRE].

[164] J.A. Harvey and M.S. Turner, Cosmological baryon and lepton number in the presence of electroweak fermion number violation, Phys. Rev. D 42 (1990) 3344 [INSPIRE].

[165] C.S. Fong, M.C. Gonzalez-Garcia and E. Nardi, Leptogenesis from Soft Supersymmetry Breaking (Soft Leptogenesis), Int. J. Mod. Phys. A 26 (2011) 3491 [arXiv:1107.5312] [INSPIRE].

[166] Planck collaboration, P.A.R. Ade et al., Planck 2013 results. XVI. Cosmological parameters, Astron. Astrophys. 571 (2014) A16 [arXiv:1303.5076] [INSPIRE].

[167] WMAP collaboration, C.L. Bennett et al., Nine-Year Wilkinson Microwave Anisotropy Probe (WMAP) Observations: Final Maps and Results, Astrophys. J. Suppl. 208 (2013) 20 [arXiv: 1212.5225] [INSPIRE].

[168] F. Capozzi, G.L. Fogli, E. Lisi, A. Marrone, D. Montanino and A. Palazzo, Status of three-neutrino oscillation parameters, circa 2013, Phys. Rev. D 89 (2014) 093018 [arXiv: 1312.2878] [INSPIRE]. 